\title{
Affect-congruent attention drives changes in reward expectations
}

\author{
Daniel Bennett ${ }^{*}{ }^{1,2}$, Angela Radulescu ${ }^{*} 3,4$, Sam Zorowitz ${ }^{1}$, Valkyrie Felso ${ }^{5}$, \\ Yael Niv ${ }^{1,3}$ \\ ${ }^{*}$ Equal contribution \\ ${ }^{1}$ Princeton Neuroscience Institute, Princeton University, USA \\ ${ }^{2}$ Department of Psychiatry, Monash University, Australia \\ ${ }^{3}$ Department of Psychology, Princeton University, USA \\ ${ }^{4}$ Center for Data Science, New York University, USA \\ ${ }^{5}$ Max Planck Institute for Intelligent Systems, Tübingen, Germany
}

\begin{abstract}
Positive and negative affective states are respectively associated with optimistic and pessimistic expectations regarding future reward. One mechanism that might underlie these affect-related expectation biases is attention to positive- versus negative-valence stimulus features (e.g., attending to the positive reviews of a restaurant versus its expensive price). Here we tested the effects of experimentally induced positive and negative affect on feature-based attention in 120 participants completing a compound-generalization task with eye-tracking. We found that participants' reward expectations for novel compound stimuli were modulated by the affect induction in an affect-congruent way: positive affect increased reward expectations for compounds, whereas negative affect decreased reward expectations. Computational modelling and eye-tracking analyses each revealed that these effects were driven by affect-congruent changes in participants' allocation of attention to high- versus low-value features of compound stimuli. These results provide mechanistic insight into a process by which affect produces biases in generalized reward expectations.
\end{abstract}




\section{Introduction}

Affect is deeply intertwined with expectations about future reward and punishment. Whereas psychological wellbeing and positive affect are linked with optimistic future expectations, negative affect is associated with pessimistic expectations (Marshall, Wortman, Kusulas, Hervig, \& Vickers, 1992; Scheier, Carver, \& Bridges, 2001). Clinically, various symptoms of mood disorders can be viewed as changes in expectation formation: mania is associated with grandiose positive expectations (S. L. Johnson, Freeman, \& Staudenmaier, 2015; Schönfelder, Langer, Schneider, \& Wessa, 2017), whereas depression and anxiety are marked by pessimistic expectations about future reward and punishment (Miranda \& Mennin, 2007; Schönfelder et al., 2017). It is therefore critical to understand how affect might influence cognitive processes that underlie expectation formation.

One process by which humans and other animals form reward expectations for novel stimuli is feature-based generalization (Rescorla, 1976; Shepard, 1987; Shanks \& Darby, 1998; McLaren \& Mackintosh, 2002). In choosing whether to visit a new restaurant, for example, a diner might consider features such as type of cuisine, the chef's reputation, and the price of meals. Using a weighted combination of these features, they can form a generalized reward expectation for the restaurant (i.e., a reward expectation formed via feature-based generalization) even before they eat there (Schulz et al., 2019).

How people form generalized reward expectations for a novel stimulus depends on how they allocate attention to its different features. For example, if our diner were to attend more to positively valenced stimulus features (i.e., features that are predictive of reward, such as a preferred style of cuisine), they would form a higher reward expectation for the restaurant than if they attended more to its negatively valenced features (such as a high price). Feature-based attention (Nosofsky, 1986; Kruschke, 1992; Niv et al., 2015; Leong, Radulescu, Daniel, DeWoskin, \& Niv, 2017) is therefore a crucial determinant of reward expectation: if attention for a stimulus is biased toward its high-value features then reward expectations will be inflated as a result, and vice versa if attention is biased towards low-value stimulus features.

Separately, human visual attention is known to be modulated by affect in an affectcongruent manner, such that subjects preferentially attend to visual stimuli that are congruent with their affective state. Whereas positive affective states facilitate attentional selection of positively valenced visual stimuli (Wadlinger \& Isaacowitz, 2006; Tamir \& Robinson, 2007; Becker \& Leinenger, 2011), negative affect is associated with increased attention to negatively valenced stimuli (Koster, De Raedt, Goeleven, Franck, \& Crombez, 2005; Koster, De Raedt, Leyman, \& De Lissnyder, 2010; Becker \& Leinenger, 2011). These results are consistent with affect-congruent information-processing biases across a number of species and cognitive domains (e.g., Bower, 1981; E. J. Johnson \& Tversky, 1983; Mayer, Gaschke, Braverman, \& Evans, 1992; Wright \& Bower, 1992; Harding, Paul, \& Mendl, 2004), though it should be noted that other, more complex patterns of interaction between affect and attention have also been demonstrated (Sedikides, 1992; Fredrickson, 1998; Moore \& Oaksford, 2002; Gable \& Harmon-Jones, 2010; Zivot, Cohen, \& Kapucu, 2013),

A striking implication of these findings is that if affective biases alter feature-based attention in the same affect-congruent manner as visual selective attention, we would 
predict affect-congruent changes in generalized reward expectations as a result. That is, we would predict positive affect to produce higher generalized reward expectations (via increased attention to high-value stimulus features), and negative affect to produce lower reward expectations (via increased attention to low-value stimulus features). This interaction between affect and attention should alter choice patterns, a hypothesis that has not previously been tested in the domain of decision-making.

To do so, we combined eye-tracking with a novel compound generalization task (Melchers, Shanks, \& Lachnit, 2008; Wagner, 2008; Soto, Gershman, \& Niv, 2014). In a learning phase, participants first learned to associate a set of simple visual cues with differing reward probabilities; then, in a generalization phase, participants were presented with choices involving novel compounds composed of pairs of simple cues from the learning phase. To test the effects of affect on reward expectation, participants experienced either a positive, neutral, or negative affect induction during the generalization phase of the task. We hypothesized that we would observe an increase (decrease) in generalized reward expectations for novel compounds following a positive (negative) affect induction, and that the mechanism for this effect would be affect-congruent changes in attention (i.e., increased attention to high-value cues in the positive affect group, and vice versa in the negative affect group).

\section{Results}

120 adult participants (77 female, 43 male; mean age $(\mathrm{SD})=21.34$ (4.40)) completed a novel compound-generalization task designed to assess the effects of affect on the distribution of attention over different cues within compound stimuli. The cover story for the task was that of a "space mining" game (see Figure 1 and Methods). Participants were told they would be mining minerals from different 'planet' stimuli, where each planet could contain either a valuable mineral (termed "positivium") or a worthless mineral ("negativium"). Different planet stimuli were marked with different cues (i.e., rune symbols), each of which was associated with a different probability of yielding the valuable mineral.

The task was divided into three phases (Figure 1A). The first phase involved learning the values of six different cues, and the second assessed whether participants had successfully learned these values. In the third phase, the critical test of our hypotheses, participants chose between compounds of the previously learned cues with no new outcomes. These generalization choice trials were interspersed with a between-participants affect-induction procedure (positive, neutral, or negative; see Methods for further details). Eye-tracking was conducted throughout all three phases of the task, and subjective current mood was elicited at the start and end of each block throughout the experiment. 


\section{A. Overview of task structure}

\begin{tabular}{|c|c|c|c|c|c|}
\hline $\begin{array}{c}\text { Simple cue } \\
\text { learning }\end{array}$ & $\begin{array}{c}\text { Simple cue } \\
\text { test } \\
\begin{array}{c}3 \text { blocks } \\
\text { of } 48 \text { trials }\end{array}\end{array}$ \\
$\begin{array}{c}1 \text { block } \\
\text { of } 30 \text { trials }\end{array}$ & $\begin{array}{c}\text { Test block 0 } \\
\text { positive/negative } \\
\text { affect groups only }\end{array}$ & Test block 1 & Test block 2 & Test block 3 \\
\hline
\end{tabular}

Eye tracking

\section{B. Trial schematic: simple cue learning}

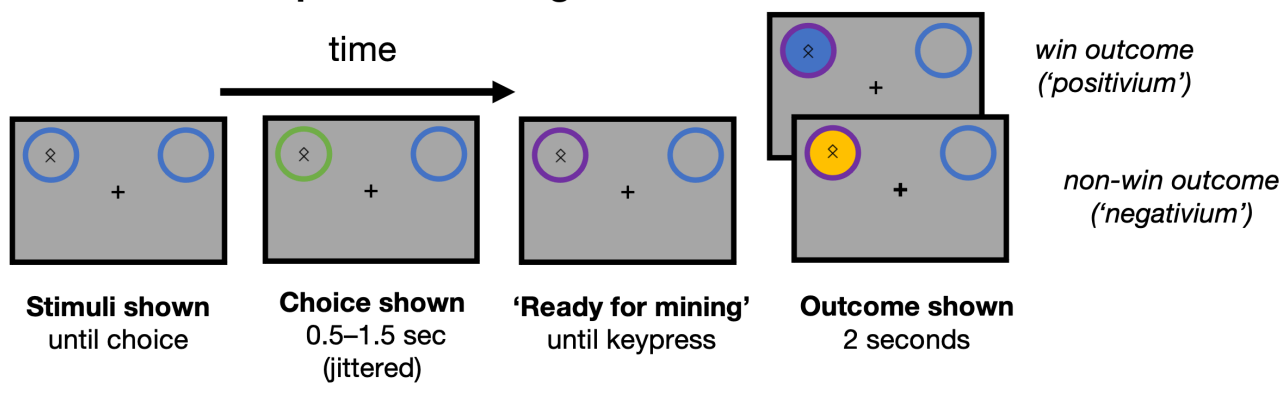

C. Trial schematic: simple cue test and compound generalization

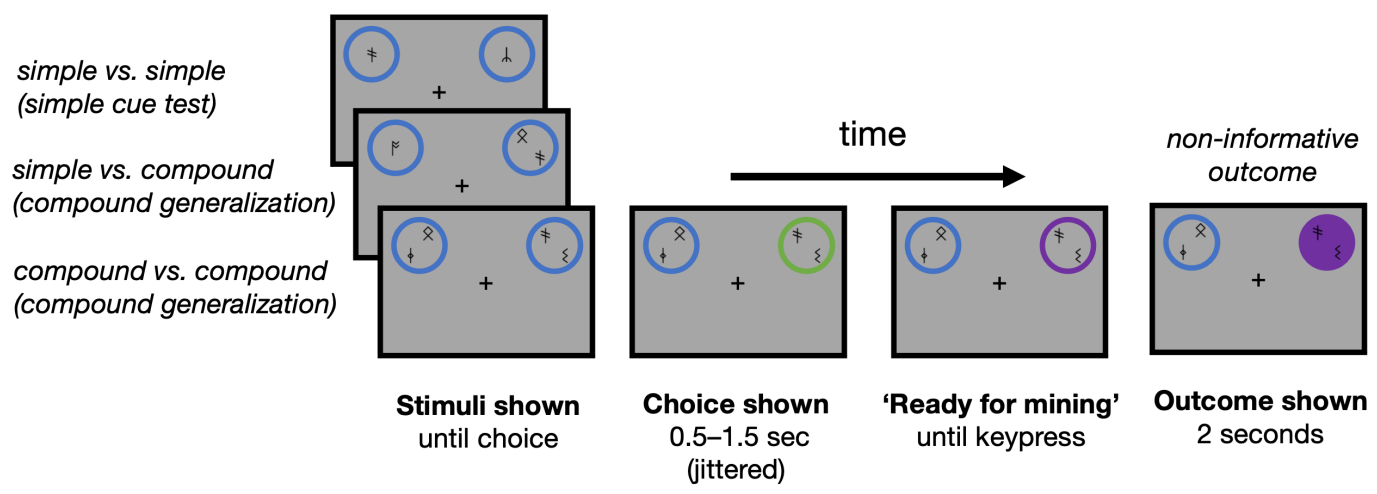

Figure 1: Task structure. A. The task comprised three phases, with affect inductions (90-second emotional film clips) presented prior to each of three blocks in the compound generalization phase. For positive/negative affect groups, the induction was preceded by one additional compound generalization block to measure generalization in neutral affect. B.: Sequence of visual events for phase 1 (simple cue learning). One cue per trial was presented to the left or right of a central fixation cross; after a "planet" was selected for "mining", its outline color changed to green to indicate the participant's selection. The chosen planet's outline color changed again to purple after a jittered interval, signifying that the outcome could now by mined. At this point, the participant could press any key to reveal the outcome, which was indicated both by a change in planet fill color to blue (win) or orange (non-win) and a distinctive win or non-win sound. C.: Sequence of visual events for task phases 2 and 3 (simple cue test and compound generalization test). The structure of each trial was the same as in phase 1, except that two stimuli were presented and the participant could choose between them, and feedback was uninformative (a neutral purple color and neutral sound). Three example cue configurations are shown, representing (from top to bottom) a simple-versus-simple choice in the simple cue test phase, a simpleversus-compound choice in the compound generalization phase, and a compound-versus-compound choice in the compound generalization phase. Cues in compound stimuli were symmetrically offset from the center of the stimulus, ensuring that the distance of all cues from fixation was approximately equal. 


\subsection{Participants accurately learned values of simple cues}

In the first phase of the task, participants learned the reward contingencies of six distinct rune cues via a Pavlovian learning procedure (Figure 1B). For each participant, there were two low-value cues (reward probability of $25 \%$; hereafter denoted $L$ ), two medium-value cues (reward probability of $50 \%$; denoted $M$ ) and two high-value cues (reward probability of $75 \%$; denoted $H$ ). In the second phase (Figure 1C), we verified that participants had successfully learned to distinguish between the different cues by offering them choices between all pairs of cues (without feedback, to avoid unequal exposure to the outcomes of each cue type due to the participant's specific choices).

Participants displayed good overall learning of cue reward probabilities (mean proportion correct in simple cue test trials $=0.89, \mathrm{SD}=0.11$ ). As expected given that the learning phase of the task preceded the subsequent between-participants affect induction, there was no evidence for a significant difference in learning between different affect induction groups $(\beta=-0.12, p=.41$; Figure $2 \mathrm{~A})$. There were also no significant associations between performance at test and individual differences in either baseline self-reported mood valence (Spearman $\rho=.05, p=.56$ ) or arousal (Spearman $\rho=.05, p=.60$ ).

We also assessed post-choice mining times (the response time for revealing the outcome after the chosen planet became ready to mine). Consistent with a general approach bias towards higher-value stimuli, mining time was significantly modulated by cue value during the simple cue test $(\beta=-0.09, p=.03)$, with a faster mining time for higher-value simple cues (mean response time [SEM] for high-value cues: $505.5 \mathrm{~ms}$ [19.3]; medium-value cues: $519.9 \mathrm{~ms}$ [21.5]; low-value cues: $548.8 \mathrm{~ms}$ [25.3]).

\subsection{Affect inductions successfully induced positive and neg- ative affect}

In the third phase of the task, participants made choices between pairs of options involving novel compounds of cues from the earlier learning phase. To measure the effects of affect on compound generalization, this phase of the task was conducted with a between-participants video-based affect induction, such that each participant saw either three positive videos, three neutral videos, or three sad videos (one video at the start of each block). Further details of affect-induction stimuli, as well as of a separate affect-induction validation experiment, are presented in the Method and in Supplementary Section S2. Participants in the positive and negative affect-induction groups also completed an additional block of compound generalization choice trials prior to the first affect induction. This enabled our computational modelling analyses of behavior (see below) to quantify the effects of the affect induction as a within-subjects change from baseline for these participants (see Methods for further details). 
A. Simple cue test accuracy

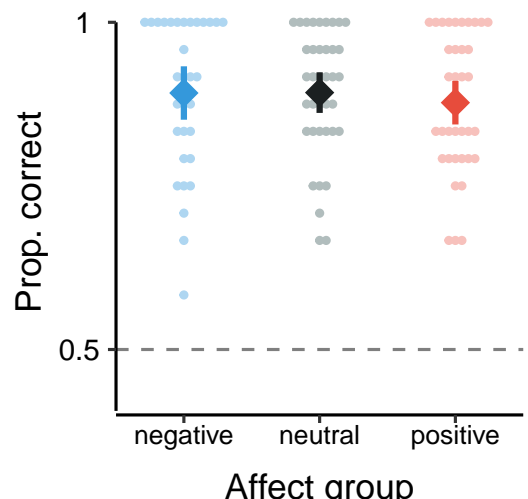

D. Compound probe trials

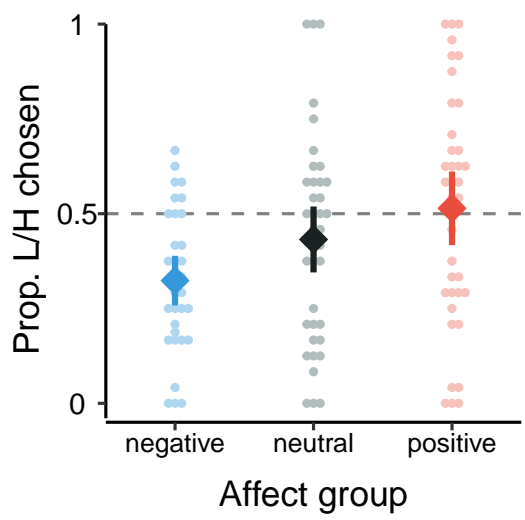

B. Affect induction

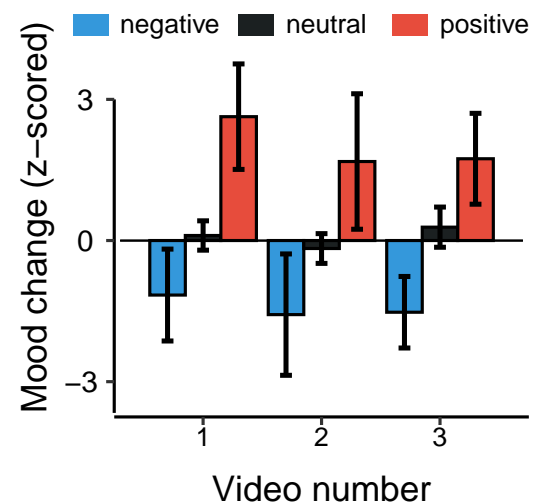

E. Simple probe trials

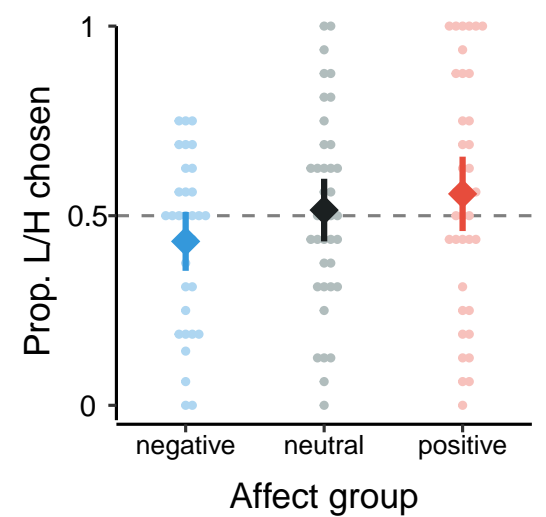

C. Generalization accuracy

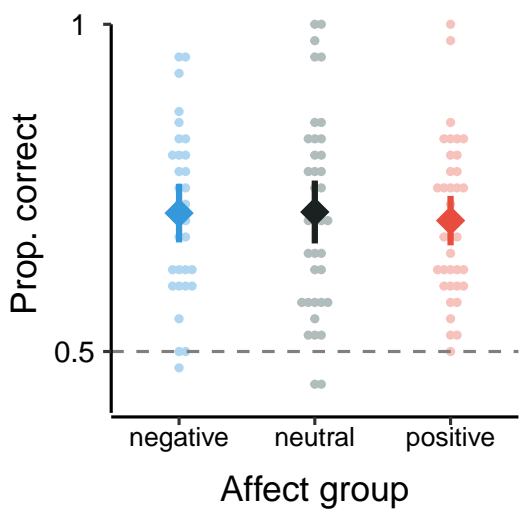

F. Probe trial consistency

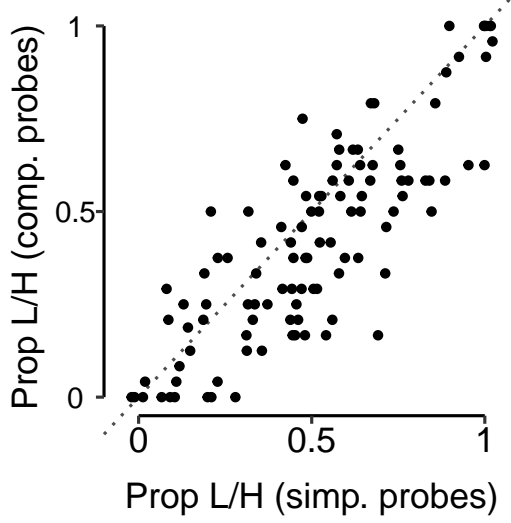

Figure 2: Behavioral results. A. The simple cue test evidenced no significant between-groups differences in choice accuracy (i.e., proportion of trials on which the higher-value cue was chosen). B. As expected, there was a significant effect of affect-induction videos on self-reported mood valence ( $\beta=2.63, p<.001$; mood reports standardized within subject with reference to the average betweenblock mood change in the learning blocks, where no videos were presented). C. In the compound generalization phase, there were no significant between-groups differences in choice accuracy (the proportion of trials in which the compound with the higher mean value was chosen). D. There was, however, a significant between-groups difference in preference for the $L / H$ stimulus as opposed to the $M / M$ stimulus in compound probe trials $(\beta=0.79, p<.001)$. E. There was also a significant between-groups difference in preference for the $L / H$ stimulus as opposed to the $M$ cue in simple probe trials $(\beta=0.56, p=.004)$. F. Preference for the $L / H$ stimulus in simple probe trials and preference for the $L / H$ stimulus in compound probe trials were positively correlated (Spearman $\rho=.81, p<.001$ ). For all panels, groups are presented as mean $\pm 95 \%$ CI. Points in scatterplots represent condition means for individual participants, and are jittered horizontally to prevent overplotting.

As expected, the affect induction significantly modulated the valence of participants' self-reported mood $(\beta=2.63, p<.001 ;$ Figure $2 \mathrm{~B})$, but did not modulate their selfreported arousal $(\beta=0.44, p=.16)$. For both valence and arousal, there was no significant effect of video number on self-reported mood change (valence: $\beta=-0.22, p=.23$; arousal: $\beta=-0.09, p=.39)$, and no significant interaction between video group and video number (valence: $\beta=-0.21, p=.34$; arousal: $\beta=-0.07, p=.60$ ), suggesting that influence of videos on self-reported mood did not change over time. 


\subsection{Participants treated compound stimulus values as weighted averages of simple cue values}

Concurrent with the affect induction, participants were presented with novel compound stimuli comprised of pairs of the simple cues that they had learned about previously (Figure 1D; see Method and Supplementary Section S1 for further information on the composition of stimulus choice pairs). Based on previous studies of compound generalization, we assumed that participants would estimate the value of a compound stimulus as a weighted average of the values of the cues within the compound (Rescorla, 1976; McLaren \& Mackintosh, 2002, one alternative to which is a non-linear conjunction rule; c.f. Saavedra, 1975; Holland, 1991). A qualitative prediction of such a linear rule is that the value of a compound stimulus should be proportional to the mean of the values of its constituent cues (e.g. an $L / H[25 / 75]$ stimulus should be treated as more valuable than an $L / M[25 / 50]$ stimulus, but less valuable than an $M / H$ [50/75] stimulus).

Figure $2 \mathrm{C}$ shows this predicted qualitative pattern in our data. In choices between compound stimuli with different mean cue values, participants tended to choose the stimulus with the higher mean value (mean choice proportion for higher-mean-value stimuli $(\mathrm{SD})=0.71(0.13) ; p<.001$, Wilcoxon test against chance). This pattern did not differ significantly across affect groups $(\beta=-0.09, p=.38)$. Furthermore, as in the simple-cue test phase, planet mining time for compound stimuli was significantly modulated by mean cue value $(\beta=-0.18, p=.04)$, such that participants were significantly faster to mine higher-mean-value compounds (mean response time [SEM] for $M / H$ compounds: 464.4 ms [16.1]; for $L / H$ or $M / M$ compounds: $470.0 \mathrm{~ms}$ [14.4]; for $L / M$ compounds: $495.5 \mathrm{~ms}$ [17.7]). These findings support the explanation that participants estimated compound cue values using a linear weighted average.

\subsection{Affect inductions produced affect-congruent biases in valuation of compound stimuli}

The primary goal of the compound generalization phase was to test participants' allocation of attention to the different simple cues contained within each compound stimulus. The main trials of interest for this analysis were 'probe trials', in which participants chose between an $L / H$ compound stimulus and either an equal-mean simple stimulus $(M$; simple probe trials) or an equal-mean compound stimulus $(M / M$; compound probe trials). In these trials, the value the participant assigned to the $L / H$ stimulus - and, therefore, their preference for this stimulus over the alternative - depended on the respective attention weights assigned to the $L$ and $H$ cues. If more attention was paid to the $L$ cue, the estimated value of the compound $L / H$ stimulus would be lower, and it would therefore tend to be chosen less frequently than the alternative stimulus (either $M$ or $M / M$ ), and vice versa if more attention was given to the $H$ cue.

Crucially, we found a clear modulation of preference for the $L / H$ stimulus by affect group, both in compound probe trials (Figure $2 \mathrm{D} ; \beta=0.79, p<.001$ ) and in simple probe trials (Figure 2E; $\beta=0.56, p=.004$ ). As we hypothesized, this modulation was consistent with affect-congruent shifts in attention such that across trial types, preference for the $L / H$ stimulus was strongest in the positive affect group, intermediate in the neutral affect 
group, and weakest in the negative affect group.

For compound probe trials, there was a significant interaction between affect group and block number, such that the effect of affect induction on choice behavior weakened over time (Supplementary Figure S8; compound probe trials: $\beta=-0.27, p=.006$; cf. simple probe trials: simple probe trials: $\beta=-0.19, p=.07)$. The main effect of block number on choice behavior was not significant for either trial type (compoound probe trials: $\beta=0.14, p=.07$; simple probe trials: $\beta=-0.08, p=.30$ ). There was no evidence for an association between any individual-difference measure and performance in either probe trial type, and no interaction between any individual difference measure and the strength of the effect of the affect induction on choice behavior (all $p>.10$ ).

Choice behavior on both simple and compound probe trials showed good internal consistency (Spearman-Brown-corrected split-half reliability: simple probe trials $\rho=.85$, compound probe trials $\rho=.88$ ). Moreover, preference for the $L / H$ stimulus was very strongly positively correlated across simple and compound probe trials (Figure 2F; Spearman $\rho=.81, p<.001)$. These psychometric results suggest that both probe trial types reliably measure the same underlying behavioral phenotype.

Lastly, we also investigated between-groups differences in overall preferences for simple versus compound stimuli over all choice pairs (Supplementary Table S1). This analysis provides a measure of whether the affect induction influenced preferences for compound stimuli in general (including but not limited to simple probe trials). Similar to the observed effects in simple probe trials alone, we found evidence for an affect-congruent modulation of preference for compound stimuli, such that preference for compound stimuli was strongest in the positive affect group, intermediate in the neutral affect group, and weakest in the negative affect group (Supplemental Figure S7A; $\beta=0.33, p=.01$ ). Once again, there was a significant interaction between this effect and block number, such that between-groups differences became less pronounced over time (Supplemental Figure S7B; $\beta=-0.15, p=.03)$. However, note that since all non-compound cues were $\mathrm{M}$ cues, simple preference for the $\mathrm{H}$ or $\mathrm{L}$ cues could manifest as preference for compound stimuli in this analysis. In our modelling of task behavior (below) we tease apart these two effects.

The interactions between affect group and block number for both simple and compound probe trials suggest that the affect induction's effect on behavior weakened over time, despite there being no block-wise differences in the effect of videos on self-reported mood. It is possible that initial surprise at the occurrence of compound stimuli might have resulted in greater overall salience of compounds in early compound generalization blocks. In this case, the greater effect of the affect induction on behavior in earlier blocks might have resulted from the fact that more attention was initially paid to these compound stimuli in absolute terms (cf. Itti \& Baldi, 2009), resulting in greater downstream effects of the affect induction on behavior.

\subsection{Computational modelling of behavior captured the ef- fects of affect on attention}

We next turned to computational modelling to dissect the influence of affect on compound generalization. We used a modelling framework (see Methods) in which the values 
of compound stimuli were assumed to be an attention-weighted linear combination of the values of simple cues within the compound (denoted $v_{j}$ ), multiplied by a parameter $\phi$ that captured the non-specific tendency for participants to prefer compound stimuli to simple stimuli or vice versa:

$$
V(\text { compound })=\phi \cdot \sum_{j=1}^{N} A_{j} \cdot v_{j}
$$

The attention weights $\left(A_{j}\right)$ for each cue in a compound were allowed to vary as a function of two cue properties: first, the relative value of each simple cue, such that attention might be biased either towards or away from high-value cues, with individual differences controlled by an $\alpha_{V}$ parameter. Second, we considered that attention might vary as a function of the certainty of cues' association with outcomes (either reward or non-reward) (Mackintosh, 1975; Pearce \& Hall, 1980), with an individual-difference parameter $\alpha_{C}$ modulating attention to cues associated with more outcome certainty. The relative influence of the two cue properties was normalised to ensure that all attention weights for a compound summed to 1 :

$$
A_{j}=\frac{\exp \left(\alpha_{V} \cdot v_{j}+\alpha_{C} \cdot\left|v_{j}-0.5\right|\right)}{\sum_{k=1}^{N} \exp \left(\alpha_{V} \cdot v_{k}+\alpha_{C} \cdot\left|v_{k}-0.5\right|\right)}
$$

Last, we assumed that choices between stimuli were made on the basis of stimulus values using a softmax policy-mapping function, with individual differences in the stochasticity of the value-to-policy mapping controlled by the inverse temperature parameter $\beta$. As such, the full framework accounted for individual differences in behavior using four parameters per participant: the compound preference parameter $\phi$, the attention-to-value parameter $\alpha_{V}$, the attention-to-certainty parameter $\alpha_{C}$, and the soft$\max$ inverse-temperature parameter $\beta$.

\subsubsection{Modelling of neutral-affect data}

First, we sought to determine whether all the above parameters were necessary to account for participants' task behavior in the absence of an affect induction. That is, we first compared the full model to simpler models that involve subsets of the 4 parameters using all choices of participants in the neutral affect group and choices of participants in the positive and negative groups in the pre-induction baseline block only.

To do this, we formulated eight models in which different subsets of the four parameters were free to vary across participants (Table 1). We fit these models to choice data using Hamiltonian Monte Carlo techniques (see Methods), and compared models using standard comparison statistics for hierarchical Bayesian models (see Methods). This approach allowed us to determine the natural dimensions of inter-individual choice variability in the task.

The results of this analysis, reported in Table 2, indicated that the full model (\#8), in which all four parameters were free to vary across participants, provided the best overall account of participants' choices in phases 2 and 3 of the task, even when accounting for (and penalizing in the model comparison) additional degrees of freedom. This suggested that each of the parameters corresponds to a meaningful dimension of individual variability in behavior. 


\begin{tabular}{ccc}
\hline Model \# & Description & $\begin{array}{c}\text { Free } \\
\text { parameters }\end{array}$ \\
\hline 1 & Baseline & $\beta$ \\
2 & Baseline with compound multiplier & $\beta, \phi$ \\
3 & Value-weighted averaging & $\beta, \alpha_{V}$ \\
4 & Certainty-weighted averaging & $\beta, \alpha_{C}$ \\
5 & Value- and certainty-weighted averaging & $\beta, \alpha_{V}, \alpha_{C}$ \\
6 & Value-weighted averaging with compound multiplier & $\beta, \phi, \alpha_{V}$ \\
7 & Certainty-weighted averaging with compound multiplier & $\beta, \phi, \alpha_{C}$ \\
8 & Value- and certainty-weighted averaging with compound & $\beta, \phi, \alpha_{V}, \alpha_{C}$ \\
\hline
\end{tabular}

Note: Simplified models (i.e., those without the full set of free parameters, \#1-7) were created by setting non-free parameters to the parameter values necessary to eliminate their effect (0 for the additive parameters $\alpha_{V}$ and $\alpha_{C} ; 1$ for the multiplicative parameter parameter $\phi$ ).

\subsubsection{Modelling the effects of the affect induction}

We next considered which of the three major parameters in Model $8\left(\alpha_{V}, \alpha_{C}\right.$, and $\left.\phi\right)$ were modulated by the affect induction ${ }^{1}$. We compared the best-fitting model from the first stage (Model \#8) to a set of more complex models in which the affect induction produced a shift in some or all model parameters (see Methods). In addition, we considered the possibility that the influence of the affect induction on model parameters decayed over time, in line with our finding that the strength of affect's influence on behavior weakened over time (Supplemental Figure S8). Unlike in the first-stage model comparison, in this stage we fit all models to all test-phase choices by all participants.

\footnotetext{
${ }^{1}$ We did not consider modulations of the softmax inverse temperature parameter $\beta$, since variation in this parameter can simply be an index of a model's overall goodness of fit.
} 
Table 2: Goodness of fit of models to neutral-affect data (model numbers as per Table 1).

\begin{tabular}{cccc}
\hline $\begin{array}{c}\text { Model } \\
\text { number }\end{array}$ & $\begin{array}{c}n \text { free parameters } \\
\text { per participant }\end{array}$ & WAIC & $\Delta$ WAIC (Std. Err.) \\
\hline 1 & 1 & 7560.19 & $1287.58(57.19)$ \\
2 & 2 & 7044.02 & $771.41(46.17)$ \\
3 & 2 & 6320.26 & $47.65(15.10)$ \\
4 & 2 & 7559.31 & $1286.70(57.19)$ \\
5 & 3 & 6316.94 & $44.33(15.00)$ \\
6 & 3 & 6276.82 & $4.21(1.99)$ \\
7 & 3 & 7040.39 & $767.78(46.25)$ \\
8 & 4 & 6272.61 & $0(-)$ \\
\hline
\end{tabular}

WAIC: Watanabe-Akaike Information Criterion, presented on a deviance scale such that lower numbers indicate better model fit. $\triangle$ WAIC: the difference between the WAIC of each model and that of the best-fitting model (model 8).

Table 3: Results of second-stage model fit to all compound generalization data.

\begin{tabular}{|c|c|c|c|c|c|}
\hline $\begin{array}{c}\text { Model } \\
\text { number }\end{array}$ & $\begin{array}{c}\text { Parameters } \\
\text { modulated by } \\
\text { affect }\end{array}$ & $\begin{array}{c}\text { Blockwise } \\
\text { decay in } \\
\text { effect }\end{array}$ & $\begin{array}{c}n \text { free } \\
\text { parameters } \\
\text { per } \\
\text { participant }\end{array}$ & WAIC & $\begin{array}{c}\Delta \text { WAIC (Std. } \\
\text { Err.) }\end{array}$ \\
\hline 8 & None & - & 4 & 13649.72 & $226.71(27.99)$ \\
\hline $8 a$ & $\phi$ & No & 5 & 13595.93 & $172.92(25.28)$ \\
\hline $8 \mathrm{a}-\lambda$ & $\phi$ & Yes & 6 & 13546.55 & $123.54(22.50)$ \\
\hline $8 \mathrm{~b}$ & $\alpha_{C}$ & No & 5 & 13647.52 & $224.51(27.99)$ \\
\hline $8 b-\lambda$ & $\alpha_{C}$ & Yes & 6 & 13651.07 & $228.06(27.97)$ \\
\hline $8 c$ & $\alpha_{V}$ & No & 5 & 13502.38 & 79.37 (19.08) \\
\hline $8 c-\lambda$ & $\alpha_{V}$ & Yes & 6 & 13457.32 & $34.31(10.61)$ \\
\hline $8 \mathrm{~d}$ & $\phi, \alpha_{C}$ & No & 6 & 13596.03 & $173.01(25.32)$ \\
\hline $8 \mathrm{~d}-\lambda$ & $\phi, \alpha_{C}$ & Yes & 7 & 13539.16 & $116.14(22.79)$ \\
\hline $8 \mathrm{e}$ & $\phi, \alpha_{V}$ & No & 6 & 13502.10 & 79.09 (18.69) \\
\hline $8 \mathrm{e}-\lambda$ & $\phi, \alpha_{V}$ & Yes & 7 & 13423.01 & $0(-)$ \\
\hline $8 f$ & $\alpha_{C}, \alpha_{V}$ & No & 6 & 13501.84 & $78.82(19.07)$ \\
\hline $8 \mathrm{f}-\lambda$ & $\alpha_{C}, \alpha_{V}$ & Yes & 7 & 13477.61 & $54.60(11.09)$ \\
\hline $8 \mathrm{~g}$ & $\phi, \alpha_{C}, \alpha_{V}$ & No & 7 & 13498.90 & $75.88(18.69)$ \\
\hline $8 g-\lambda$ & $\phi, \alpha_{C}, \alpha_{V}$ & Yes & 8 & 13426.41 & $3.39(2.80)$ \\
\hline
\end{tabular}

WAIC: Watanabe-Akaike Information Criterion, presented on a deviance scale such that lower numbers indicate better model fit. $\triangle$ WAIC: the difference between the WAIC of each model and that of the best-fitting model (model $8 \mathrm{e}-\lambda$ ). 
The results of this model comparison (Table 3) supported a model in which affect induction influenced two of the three parameters of interest: $\alpha_{V}$, which controls attention to value, and $\phi$, which controls the preference for compound relative to simple cues. In addition, in the best-fitting model, the strength of affect's influence on these parameters decayed over successive compound generalization blocks (decaying according to parameter $\lambda$ ). We note again that this block-wise change in parameters cannot be attributed to influences of affect on learning, since the compound generalization phase of the task did not involve feedback, and therefore did not prompt new learning.

Our model fitting assumed that participant-level parameters were drawn from grouplevel distributions and estimated the means and standard deviations of these distributions from the data. Figure 3 presents samples from the posterior (i.e., post-fit) distributions for the group means of several of these parameters (means of affect-independent parameters in grey, mean affect-related changes in parameters in purple). The mean of the distribution over the value-attention parameter $\alpha_{V}$ was negative (though overlapping with zero, see Figure 3A; SD of group distribution =3.12). Negative values of $\alpha_{V}$ mean that higher values of a cue decreased attention to that cue. The group mean for the affect-related change in this parameter $\left(\Delta \alpha_{V}\right.$; Figure 3D) was credibly greater than zero (posterior median $=0.72,95 \%$ Bayesian Highest Density Interval (HDI) of $[0.01,1.56]$ ), indicating affect-congruent modulation of attention to value, consistent with the probe-trial results presented in Figure 2D and E. That is, as positive affect increased, so did attention to higher valued cues.

The group mean of the $\phi$ parameter was significantly above one (Figure 3B; SD for group distribution $=0.09$ ), indicating an overall preference for compound stimuli rather than those including only one cue. The change in this parameter due to affect, $\Delta \phi$, was numerically negative overall, but was not credibly different from zero (posterior median $=-0.03,95 \%$ HDI [-0.08, 0.02]; Figure 3E), suggesting that positive/negative affect did not alter overall preference for compound stimuli significantly.

The group mean of $\alpha_{c}$, the attention to certainty parameter, was zero-centered (Figure $3 \mathrm{C}$; $\mathrm{SD}$ of group distribution $=3.78)$, suggesting that there was not a general tendency to attend more to the more certain $(25 \%$ and $75 \%)$ or more uncertain $(50 \%)$ cues across the participant sample.

Finally, the posterior distribution of mean of the decay parameter $\lambda$ was significantly smaller than one (posterior median $=0.44,95 \%$ HDI $[0.1,0.88]$; Figure 3F), suggesting a considerable block-by-block reduction in the strength of affect's influence on behavior. 


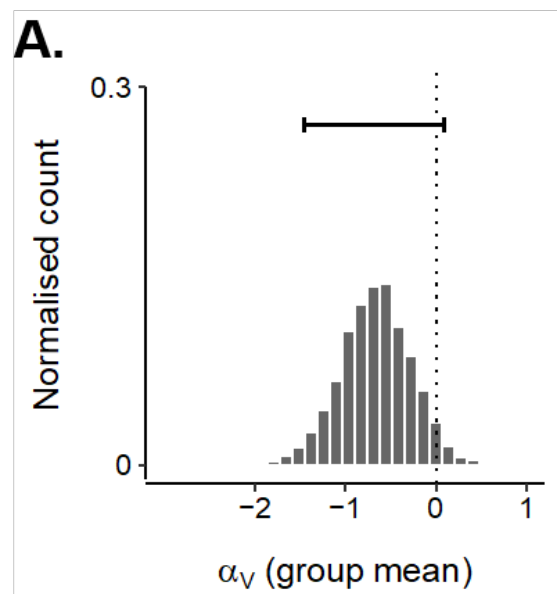

B.

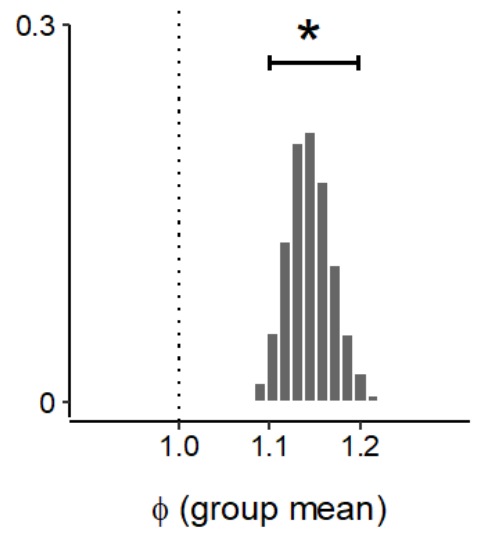

E.

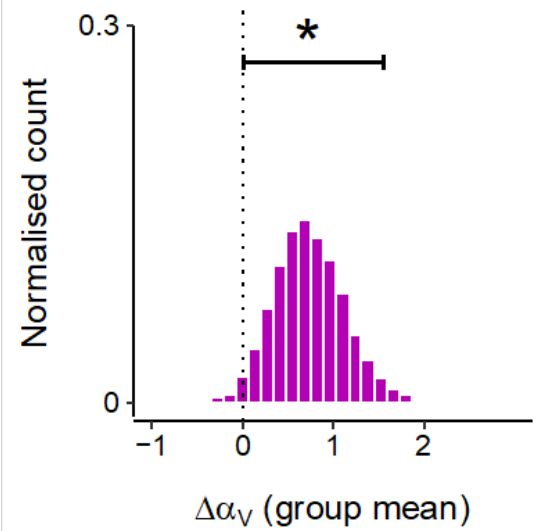

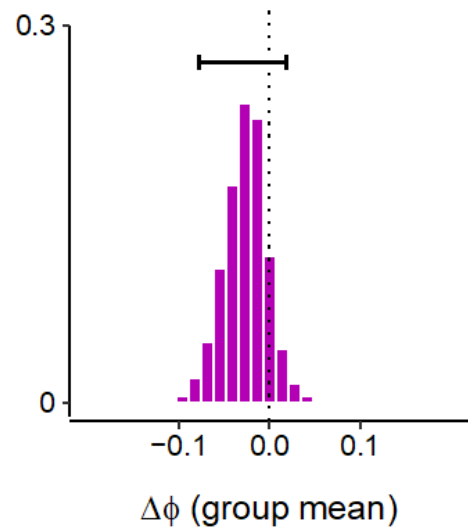

C.

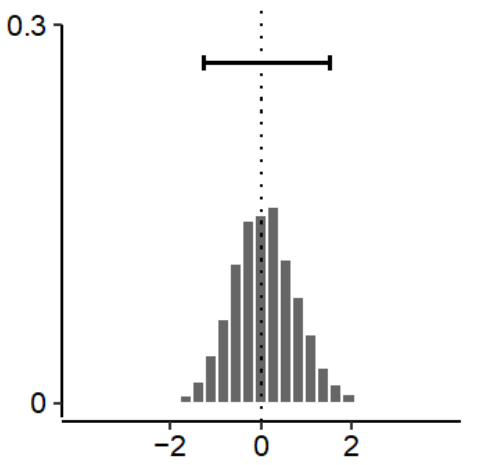

$\alpha_{C}$ (group mean)

F.

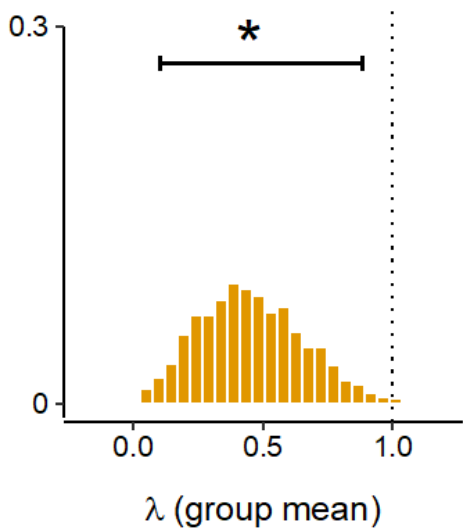

Figure 3: Estimated posterior distributions for group-mean parameters. Top histograms: group-level means for the parameters $\alpha_{V}(\mathrm{~A}), \phi(\mathrm{B})$, and $\alpha_{C}(\mathrm{C})$. Bottom histograms: group level means for the effect of affect on $\alpha_{V}(\mathrm{D})$, the effect of affect on $\phi(\mathrm{E})$, and the block-wise decay in the strength of these effects, $\lambda(\mathrm{F})$. Horizontal error bars denote the $95 \%$ credible interval for each parameter, and vertical dotted lines denote the reference value for each parameter (i.e., the parameter value at which there is no influence of the parameter on behavior: 0 for additive parameters, 1 for parameters that were multiplicative or exponents). Asterisks denote parameters for which the $95 \%$ credible interval does not include the reference value.

To aid interpretation of these parameter values, Figure 4 illustrates the dynamics of the $\alpha_{V}$ parameter in the model for different affect groups across time. As mentioned, the negative value of $\alpha_{V}$ at a group level indicates that in the neutral group, participants tended to attend more to low-value cues rather than high-value cues. However, this tendency was modulated by the affect induction in an affect-congruent fashion: participants in the negative-affect group tended to attend even more strongly to low-value cues than participants in the neutral group. By contrast, the effect of the positive affect induction was to mitigate the baseline tendency to attend more strongly to low-value cues. The effect of the affect inductions tended to diminish over time, however, such that by the final block of the task, attention to value did not differ strongly across the affect-induction groups.

Note that our modelling approach, in which affect group was coded as a continuous 
predictor, made an important additional assumption: our models implicitly assumed that the effects of positive and negative affect inductions on model parameters were equal in magnitude but opposite in sign. There is no reason why this must be the case (for instance, for a given parameter it is entirely feasible that only the positive affect induction influenced the parameter, but that the negative affect induction had no effect).We therefore conducted an additional round of model comparisons to test this assumption directly (see Supplementary Section S9). The results of these additional analyses supported the idea that effects of positive and negative affect were symmetric and in opposite directions.

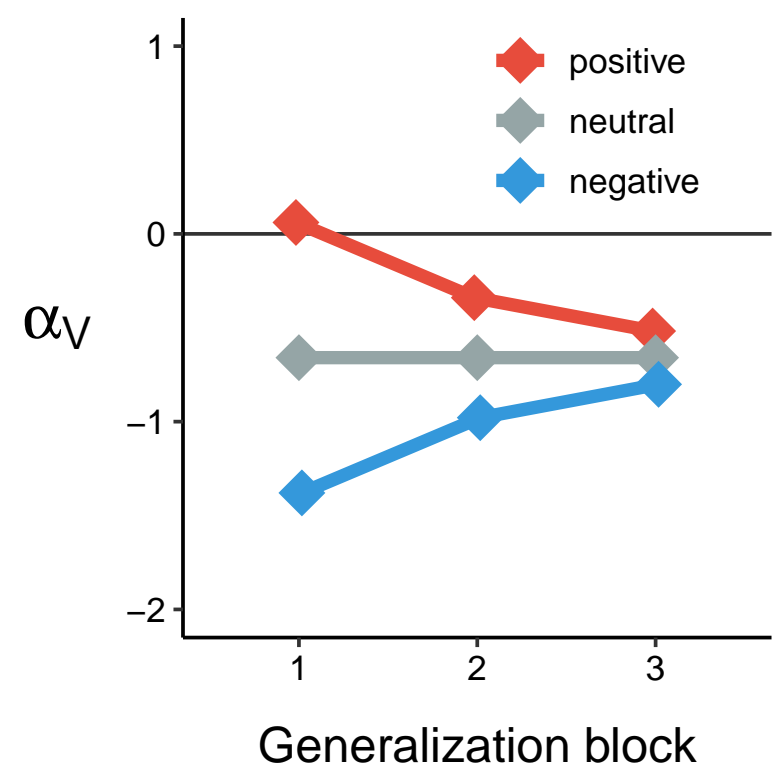

Figure 4: Illustration of model estimates of attention to valence across time and between groups. Negative values of $\alpha_{V}$ indicate more attention to low-value cues; positive values indicate more attention to high-value cues. There was a strong affect-congruent modulation of attention to value in the generalization blocks, the strength of which decayed over time. The dynamics of mood as plotted here were calculated using medians of the posterior distributions of the $\alpha_{V}, \Delta \alpha_{V}$, and $\lambda$ parameters.

\subsection{Eye-tracking data are consistent with affect-congruent modulation of attention}

In the analyses above, we interpreted compound-generalization choice behavior in terms of the allocation of attention to different simple cues within compounds. To further validate differences in behavior specifically in terms of attention and not other cognitive processes which could, in principle, cause similar effects, we used eye-tracking data $(\mathrm{N}=33$, $\mathrm{N}=32$ and $\mathrm{N}=24$ participants in the neutral, positive and negative groups respectively). We tested whether affect-congruent modulations of behavior in the compound generalization task were accompanied by changes in overt attention as measured by patterns of visual fixations.

To validate our eye-tracking measure, we first tested whether eye-gaze in the simple versus simple trials of the cue test replicated previous findings (Krajbich, Armel, \& 
Rangel, 2010; Krajbich \& Rangel, 2011; Callaway \& Griffiths, 2019). We asked whether participants' gaze was biased towards to-be-chosen stimuli, as predicted by models that posit a sequential sampling mechanism whereby participants choose an option once they gather enough evidence to compute its expected value. Indeed, even before making a choice, participants were more likely to fixate on the to-be-chosen stimulus (Figure 5A; main effect of subsequent choice status, $F(1,86)=19.94, p<.001, \eta_{p}^{2}=0.19$; no significant interaction between choice and affect group, as expected given that this choice test preceded the affect manipulation). We then tested whether attention was biased towards cues in proportion to their value. We found that relative looking time increased as a function of cue value, consistent with the prediction that people spend more time looking at cues associated with higher values (Figure 5B; main effect of cue value, $F(1,86)=31.84, p<.001, \eta_{p}^{2}=0.27$, no significant interaction $)$. 
A.

c.

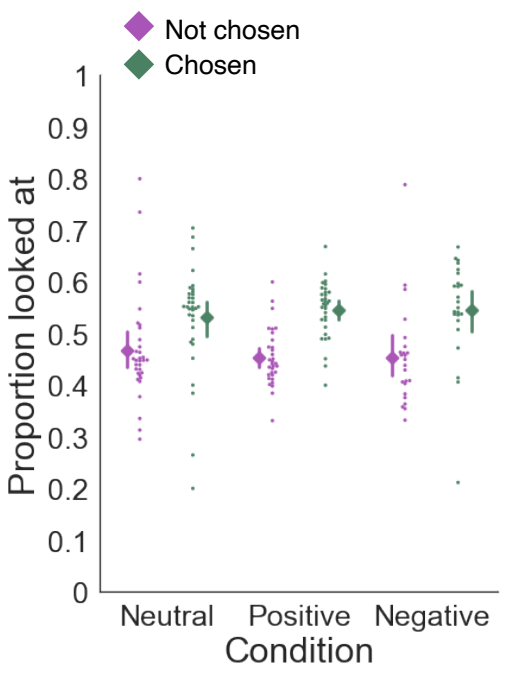

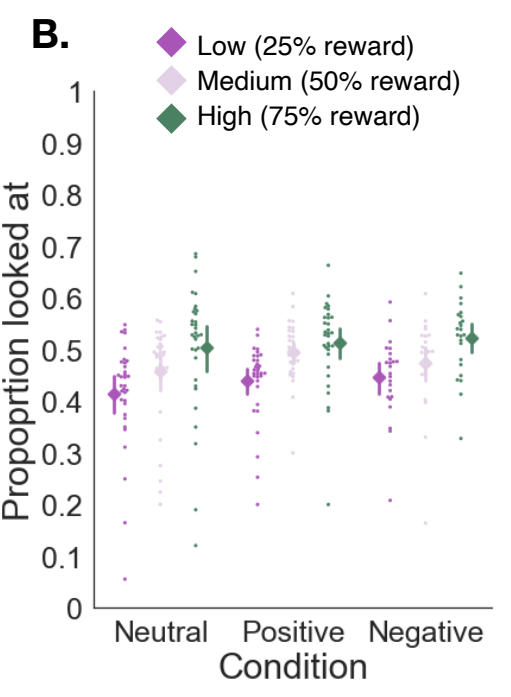

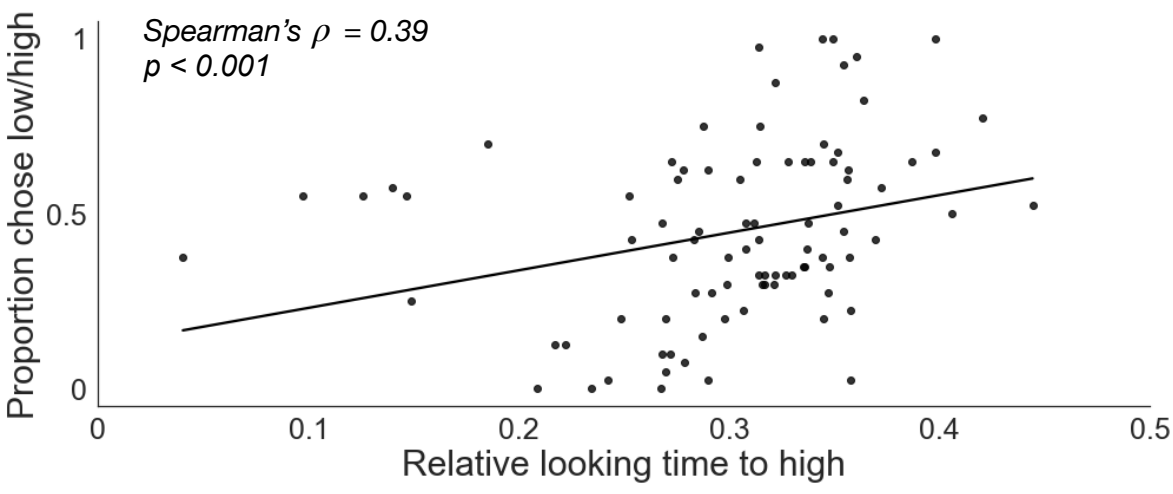

Figure 5: Eye-tracking validation results. A: Proportion of time spent looking at each cue in simple versus simple trials, as a function of whether a cue was subsequently chosen or not. For all groups, participants tended to look more at the to-be-chosen cue. B: Proportion of time spent looking at each cue in simple versus simple trials, as a function of cue value $(25,50$ or $75 \%$ probability of reward). For all groups, participants looked longer at more valuable cues. Diamond markers denote the mean of each group and its $95 \%$ confidence interval; dots indicate individual participants. C: Relative looking time to the high-valued cue within a low/high compound (that is, normalized to the total time looking at that compound stimulus), plotted against the tendency to choose the low/high compound in probe trials. The longer participants looked at the high-value cue within the low/high compound, the more likely they were to choose the low/high compound over a medium or medium/medium stimulus of the same expected value.

While value-based attention measured with eye-tracking has mainly been investigated in simple choice, our main goal was to use eye-tracking to probe changes in attention during compound generalization (Leong, Radulescu, et al., 2017). To this end, we tested whether, in probe trials, time looking at the high-value cue within an $L / H$ compound stimulus predicted choice of that stimulus. Indeed, we found that the more time people spent looking at the high-value cue relative to time looking at all other cues in the trial, the more likely they were to choose the $L / H$ stimulus over a $M$ or $M / M$ stimulus of the same expected value (Figure $5 \mathrm{C}$, Spearman $\rho=.39, p<.001$ ). 


\section{A. Compound probe trials}

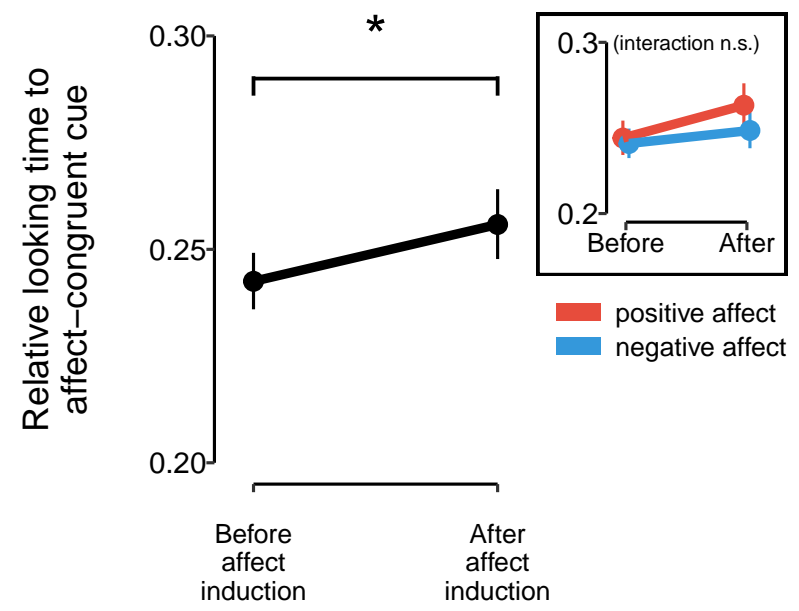

\section{B. Simple probe trials}

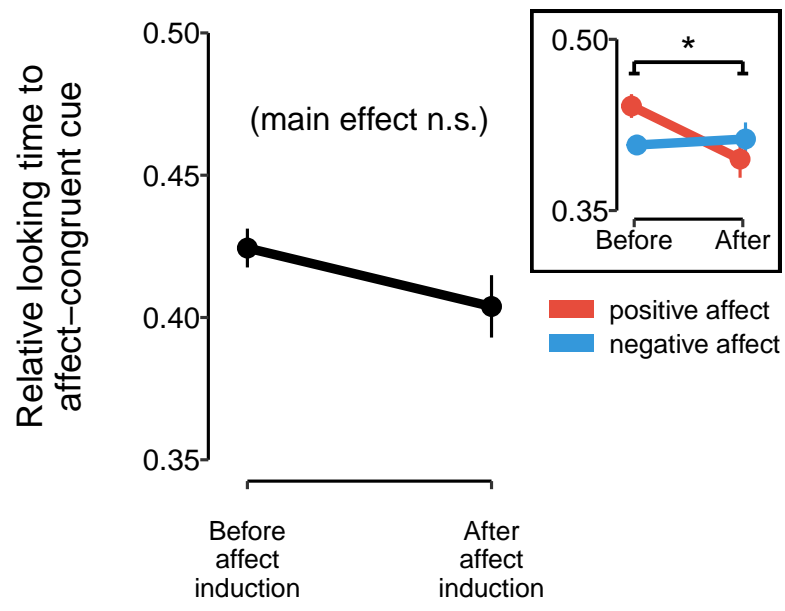

Figure 6: Affective modulation of relative looking time. Average relative looking time to the affect-congruent cue (i.e., the low-value $25 \%$ cue for participants receiving a negative affect induction and the high-value $75 \%$ cue for participants receiving a positive affect induction) as a function of timepoint (pre- versus post-affect induction). Data are plotted separately for compound probe trials (A) and simple probe trials (B); there was a significant increase in time looking at affect-congruent cues after the affect induction in compound probe trials (main effect of time; $p<.05$, denoted by *), but not in simple probe trials. Inset: data separated by affect-induction condition (red: positive affect; blue: negative affect). The affect by timepoint interaction was significant for simple probe trials $(p<.05)$ but not for compound probe trials. Error bars denote the standard error of the mean, and were computed based on estimated marginal means from the linear mixed-effects analysis.

Based on the results of the computational modelling analyses, we directly investigated the role of positive and negative affect in driving overt attention by assessing how relative looking time to affect-congruent cues changed following the affect induction. In this analysis, we simultaneously quantified the extent to which the positive affect induction increased attention for the high-value $75 \%$ cue, and the negative affect induction increased attention to the low-value $25 \%$ cue. A mixed-effects model predicting looking time to affect-congruent cues within the $L / H$ compound relative to time looking at all other cues in the trial showed a significant interaction between probe type (simple vs. compound) and affect induction (pre- vs. post-induction; $\chi^{2}(1)=5.15, p=.02$ ). Post-hoc tests revealed that this interaction was driven by a significant increase in time looking at affect-congruent cues in compound probe trials $(\beta=0.071, p=.03$; Figure $6 \mathrm{~A})$ but not in simple probe trials $(\beta=-0.073, p=.19$; Figure $6 \mathrm{~B})$. That is, when participants made a choice between a $L / H$ and a $M / M$ compound, we found evidence that participants spent more time looking at high-value cues following a positive affect induction, and more time looking at low-value cues following a negative affect induction. This finding is consistent with the results of the computational modelling analyses, in which we found that the affect induction specifically altered the $\alpha_{V}$ parameter of the model, which modulates attention to high- versus low-value cues.

Separately, we also found evidence for a significant three-way interaction $\left(\chi^{2}(1)=\right.$ $3.95, p=0.047$; see insets in Figure 6) between probe type, timepoint (before versus after 
the affect induction) and affect induction valence (positive versus negative). Post-hoc tests revealed that this interaction was largely driven by a difference in time looking at affectcongruent cues in simple probe trials prior to the affect induction $(\beta=-0.07, p=.01)$. Since this pre-induction difference is an effect of no interest and did not influence the primary effects of interest within the compound probe trials as described above, we do not interpret this finding further here.

\section{Discussion}

In this study, we tested the influence of positive and negative affect on participants' behavior in a novel compound generalization task. We hypothesized that affect would alter reward expectations for unfamiliar compound stimuli via affect-congruent modulation of attention to different features of the compounds. As predicted, we found that participants receiving a positive affect induction showed increased reward expectations for compound stimuli relative to those receiving a neutral affect induction, and vice versa for participants receiving a negative affect induction. Crucially, computational modelling suggested that this effect was driven by affect-congruent changes in the allocation of participants' attention to high-value versus low-value cues within compound stimuli. These results were corroborated by analyses of participants' gaze-fixation patterns, which revealed an analogous pattern of affect-congruent modulation of overt attention to highversus low-value cues.

Taken together, these results suggest a potential computational and perceptual-level mechanism for the well-documented finding that positive affect is associated with optimistic future expectations (i.e., value), whereas negative affect is linked with pessimistic future expectations (Erez \& Isen, 2002; Paul \& Pourtois, 2017; Scheier et al., 2001; Marshall et al., 1992). Concretely, systematic affect-congruent biases in attention to highversus low-value features of abstract future prospects (e.g., attending to positive reviews of a fine-dining restaurant in a positive mood, versus attention to its large expected expense in a negative mood) would be expected to produce increased reward expectancies in positive affective states and lowered reward expectancies in negative affective states. This is consistent with previous studies showing an increased perceptual attention bias towards positive stimuli in individuals that score high on trait optimism, happiness, and pronenness to hypomania (Segerstrom, 2001; Raila, Scholl, \& Gruber, 2015; Gruber et al., 2021). By extension, our results may also suggest a potential mechanism for affectcongruent changes in reward and punishment expectations in mania, depression, and anxiety (S. L. Johnson, 2005; A. K. MacLeod, 1996; A. K. MacLeod, Tata, Kentish, Carroll, \& Hunter, 1997; Rief et al., 2015).

Notably, our eye-tracking results produced an unexpected pattern of results when trials were subdivided into simple probe trials (involving choices between one singlecue stimulus and one compound-cue stimulus) versus compound probe trials (involving choices between two distinct compound stimuli). We found that, consistent with the behavioral and computational modelling results, relative time looking at affect-congruent cues (i.e., looking time for high-value cues after a positive affect induction and for lowvalue cues after a negative affect induction) increased as a result of the affect induction. 
This effect was driven by compound probe trials, but was not present in simple probe trials. One potential explanation for this unexpected distinction is that compound stimuli pose a more stringent requirement for overt attention (i.e., shift of eye gaze) due to visual crowding (Whitney \& Levi, 2011), whereas simple stimuli are more readily processed using covert attention only (see, e.g., Poder, 2006; Vater, Kredel, \& Hossner, 2017). As a result, our eye-tracking measure may be a more accurate assessment of attention in the compound probe trials, where both stimuli were compounds.

Independent of the affect induction, we found that in our setting, participants tended to evaluate compound stimuli as linear combinations of the values of their constituent cues. Although linear cue combination is a common assumption across models of generalization (e.g., Rescorla \& Wagner, 1972; Pearce, 1987), we note here that participants were never instructed regarding the meaning of compound stimuli, and never experienced outcomes associated with these stimuli. As such, they could conceivably have chosen other strategies, such as evaluating the compound using a non-linear cue combination rule, as is observed in the biconditional discrimination paradigm (Saavedra, 1975).

Within the set of possible linear cue combination rules, we found that participants' behavior on compound generalization was more consistent with cue-averaging than with cue-summation. This is notable because cue averaging is predicted by configural models of compound generalization (e.g., Pearce, 1987), which assume that compounds are treated as unique configurations that accrue value according to their similarity to the values of other stimuli (the simple cues, in this case). By contrast, elemental models of compound generalization assume that expectations for compound stimuli are computed by summing the values of independent features (Rescorla \& Wagner, 1972; Melchers et al., 2008; Duncan, Doll, Daw, \& Shohamy, 2018; Radulescu, Niv, \& Ballard, 2019). Here, elemental models would predict that participants would never prefer simple stimuli over compound stimuli (since the summed value of compound cues was always equal to or greater than the value of simple cues in our task). More broadly, whether behavior is consistent with elemental or configural generalization has been shown to depend on experimental context (for review see Soto et al., 2014; Kehoe, Horne, Horne, \& Macrae, 1994). In our case, the cover story, which suggested that probabilities of outcome could change across planets, but not the amount of outcome available, likely favored configural (linear combination) generalization over elemental summation. Our model predicts an influence of affect on generalized reward expectations for both configural and elemental generalization. Further research is needed to test our hypothesis in the elemental case, for instance by testing the effects of affect on generalization for compounds comprising cues from different sensory modalities, or comprising cues associated with different outcome amounts rather than different outcome probabilities.

In our computational model, the influence of each cue within a compound on reward expectations was dynamically weighted by attention (Leong, Radulescu, et al., 2017). Attention provides a mechanism for changing the degree of generalization from each simple cue to the compound, and our results suggest that affect-congruent attention may play an important role in how reward expectations generalize across features. However, affect-congruence is not the only principle that might underlie affective modulation of attention. One influential alternative model suggests that positive affect broadens the scope 
of attention (Fredrickson, 2004). Affect-related broadening might lead to generalization of reward expectations across a broader set of features, similar to the kind of temporal generalization predicted by several recent normative models of affect (Eldar, Rutledge, Dolan, \& Niv, 2016; Zorowitz, Momennejad, \& Daw, 2020). Our results for simple probe trials are consistent with this hypothesis, given that we observed a more equal allocation of attention across cues in a compound in the positive-affect condition relative to the negative-affect condition. More broadly, reward generalization is a promising transdiagnostic computational construct that warrants further investigation as an explanatory computational model of affective disorders (Radulescu \& Niv, 2019; Bennett, Silverstein, \& Niv, 2019).

Our findings are also in line with a separate body of research studying affective biases in humans and other animals (Harding et al., 2004; Mendl, Burman, Parker, \& Paul, 2009; Daniel-Watanabe, McLaughlin, Gormley, \& Robinson, 2020; Aylward, Hales, Robinson, \& Robinson, 2020). In these studies, participants are trained with an excitatory and an inhibitory conditioned stimulus that differ from one another along one perceptual dimension (e.g., high-frequency vs. low-frequency audio tones). Affective biases are measured in terms of behavioral responses to subsequent generalization stimuli intermediate between the two training stimuli; across species, positive affect has been associated with an increased positivity bias (i.e., increased generalization of the excitatory conditioned stimulus to intermediate stimuli), and vice versa for negative affect (Harding et al., 2004; Mendl et al., 2009; Daniel-Watanabe et al., 2020; Aylward et al., 2020). Our results suggest a potential interpretation of these findings in terms of internal attention to appetitive versus aversive internal representations of stimuli (see Chun, Golomb, \& Turk-Browne, 2011). Indeed, by reconceptualising interpretation biases as arising from the allocation of attention between latent dimensions of reward-predictive stimuli, affect-congruent attention biases may provide a unifying explanation for effects of affect on judgement and interpretation more broadly (e.g., C. MacLeod, Mathews, \& Tata, 1986; Eysenck, Mogg, May, Richards, \& Mathews, 1991; Hirsch \& Mathews, 1997).

The modulation of attention by affect is a phenomenon that presents a challenge to many formal computational models of affect. Ransom et al. (2020) recently showed that this is particularly the case for predictive-processing models of affect (e.g., Joffily \& Coricelli, 2013), which conceptualise attention as being driven by the expected precision of sensory inputs, rather than by affective factors. Similarly, although less challenging to core tenets of the theory, affective modulation of attention is also not predicted by recent reinforcement-learning models of affect (Eldar et al., 2016; Bennett, Davidson, \& Niv, 2021). Our findings demonstrate one manner in which reinforcement-learning models might be extended to account for affective modulation of attention in real-world settings where stimuli and outcomes have multiple features that must be attended to. Further research is required to integrate these ideas into normative accounts of affect, though some preliminary work suggests the form that such a theory might take (Vinckier, Rigoux, Oudiette, \& Pessiglione, 2018; Radulescu \& Niv, 2019; Bennett \& Niv, 2020; Eldar, Pessiglione, \& van Dillen, 2021).

Finally, our results also have implications for computational models of value-based attention (Anderson, Laurent, \& Yantis, 2012). Sampling-based models have suggested that 
attentional effects during value computation, and people's overt fixation patterns, reflect evidence accumulation over internal beliefs about the probability of reward (Krajbich et al., 2010; Krajbich \& Rangel, 2011; Krajbich, Lu, Camerer, \& Rangel, 2012; Callaway \& Griffiths, 2019). The results of our study suggest that fixation patterns during decisionmaking are sensitive to affect. Sampling models suggest potentially wide ramifications of this finding: if affect biases the extent to which positive or negative experiences are integrated into a value signal by influencing what people attend to both in the external environment and internally in memory, that can explain tendencies to expect low values in disorders such as depression (cf. Bower, 1981; Rouhani \& Niv, 2019). In general, formalising how affective biases arise from information sampling may help integrate empirical findings suggesting that both attention and memory are biased by affect, and in turn mediate affect's downstream effects on choice (Gotlib \& Joormann, 2010; Raila et al., 2015).

To conclude, we provide direct evidence from behavioral and eye-tracking data that experimentally induced changes in affect bias attention towards features predictive of affect-congruent reward outcomes. That is, positive affect leads to increased attention to high-value stimulus features, whereas negative mood produces increased attention to low-value features prospects. These results suggest a potential cognitive mechanism for affect-congruent modulation of future reward expectations in the general population, as well as for extreme forms of affect-congruent changes in reward expectations associated with psychiatric syndromes including mania, hypomania, and depression.

\section{Method}

\subsection{Participants}

We recruited a total of 120 participants (77 female, 43 male; mean age [SD] $=21.34$ [4.40], age range 18 to 55), via online advertisements from the general population in Princeton, New Jersey, USA. This study was approved by the Institutional Review Board of Princeton University, and all participants provided written informed consent and had normal or corrected-to-normal vision. Total study duration was approximately 90 minutes per participant. Participants received monetary compensation for their time and travel expenses, plus an incentive-compatible bonus for task performance (mean payment = USD $\$ 19.01, \mathrm{SD}=2.92$ ).

\subsection{Design}

Participants were allocated to one of three affect groups (positive, neutral, or negative, 40 participants in each group; see Affect induction procedure below) in a randomized between-subjects design. This sample size was designed to give in excess of $80 \%$ power to detect a moderate effect size of $f^{2}=0.3$ (under standard assumptions of the general linear model, and with $\alpha=.05)$. Participants completed a behavioral task with concurrent eyetracking recording, as well as self-report measures of trait depression and hypomania (the General Behavior Inventory (GBI); Depue et al., 1981) and current positive and negative affect (the Positive and Negative Affect Schedule (PANAS); Watson, Clark, \& 
Tellegen, 1988). To ensure that self-report measures were not influenced by the affect induction that occurred during the behavioral task, self-report measures were presented before the task, with the order of the two self-report instruments counterbalanced across participants.

\subsection{Behavioral task}

The cover story for the behavioral task was a "space mining game" (see Figure 1). Participants were instructed that they would mine minerals from different "planets", and that each planet stimulus could be made of a valuable mineral or a worthless mineral (differentiated by color, blue vs. yellow). Different planet stimuli were marked with different cues (i.e., rune symbols), and participants were informed that each cue or combination of cues provided information about the probability that a given planet would yield the valuable mineral when mined. In order to incentivize learning, participants received a monetary bonus proportional to the amount of the valuable mineral that they mined across the task.

The task comprised three phases: an initial cue learning phase, a learning test involving choices between different cues, and a compound generalization phase involving planet stimuli marked with multiple cues ('compound stimuli'). On each trial, two planet stimuli were presented: one above and to the left of a central fixation cross, and the other above and to the right and participants chose one of the two planets to "mine" using the computer keyboard (left/right arrow keys). After a short delay (0.5 to 1.5 seconds, uniformly jittered), the chosen planet became "available to mine" (represented visually by a change in the its border color) and participants could press and key to extract the mineral on the planet. Each trial therefore yielded 3 measures: stimulus choice, choice response time, and reaction time for mining the planet (i.e., time elapsed between when the planet become ready to mine and when the participant mined it). Mining reaction time was taken as an index of approach motivation for the chosen stimulus.

Stimuli were presented in MATLAB with PsychToolbox (Brainard, 1997), using a CRT monitor at a resolution of $1024 \times 768$ pixels. Participants were seated comfortably using a chin rest at a distance of $57 \mathrm{~cm}$ from the screen, such that $1 \mathrm{~cm}$ on the screen subtended a visual angle of 1 degree. The radius of each planet stimulus was 2.5 centimetres, and each was diagonally offset 13 centimetres from a central fixation cross. Audio was presented via headphones.

\subsubsection{Learning phases}

In the first phase of the task, participants learned to associate six cues with different reward probabilities (24 exposures per cue randomly intermixed across 3 blocks of 48 trials each). Two cues were associated with a low probability of reward (25\%), two with a medium reward probability (50\%), and two with a high reward probability $(75 \%)$. The allocation of cue images to reward probabilities was randomized across participants. On each trial, a single cue was presented in the center of either the left planet stimulus or the right planet stimulus. Participants were informed that planets without a cue ("empty planets") had zero probability of yielding the valuable mineral, and that it would not 
benefit them to choose these stimuli. Consistent with this instruction, participants very infrequently chose planets without a cue (mean number of empty-planet choices across participants $=2.7$, out of a total of 144 first-phase trials). One participant from the negative-affect group who chose the empty planet on more than $25 \%$ of learning trials was excluded from further analysis.

The second phase of the task assessed the learning of cue-reward contingencies. Participants completed a single block of choices between the six cues whose reward probability was learned in phase 1 (30 trials total; two repetitions of each possible cue pair). This allowed us to verify that participants had indeed learned to discriminate between cues. To ensure that no further learning took place in this task phase, no informative feedback regarding the outcome of participants' choices was provided. Participants were instructed that their selections would still count towards the monetary bonus that they received at the end of the experiment, but that they would not receive trial-by-trial information on the outcomes of their choices (see Figure 1C). 7 participants (five from the negative-affect group and one each from the positive-affect and neutral-affect groups) who did not display above-chance performance levels in this phase of the task (as determined by a binomial test against chance; $\alpha=.05$, one-tailed) were excluded from further analysis.

\subsubsection{Compound generalization phase}

The third phase of the task assessed compound generalization across three blocks of 32 trials each. This phase introduced compound stimuli containing two rune cues rather than one (see Figure 1C). As in the simple-cue test phase, no feedback was provided on the outcome of participants' choices. Compound stimuli were introduced into the task without any additional instructions to participants on how they ought to be interpreted: as in the second phase of the task, participants were informed that their choices would continue to count towards their monetary bonus (though they were not given any information on how the value of compound stimuli would be assessed), despite the lack of outcome feedback. By withholding informative feedback on participants' choices we ensured that we were assessing participants' generalization from previous learning, distinct from new learning of the value of the compounds themselves. This presents a contrast with previous studies of attention to compound stimuli (see Kruschke, 2001; Le Pelley, Mitchell, Beesley, George, \& Wills, 2016, for review), which studied the distribution of attention at learning rather than at choice.

We focused our analysis on two kinds of choice trials that were expected to be especially diagnostic of the distribution of attention within compounds. In simple probe trials (16 of 96 trials in compound generalization phase) participants chose between a medium-valued simple stimulus (i.e., a planet marked with a single $50 \%$ reward cue) and a low/high compound stimulus (a planet marked with one $25 \%$ reward cue and one $75 \%$ reward cue). Second, in compound probe trials (24 trials) participants chose between a medium/medium compound and a low/high compound.

These probe trials provide a critical test of the distribution of attention over cues in a compound. If the participant divides attention equally among the cues in a compound stimulus, then a low/high compound stimulus has the same expected value as both a medium-value simple stimulus and a medium/medium compound. If the participant al- 
locates more attention to the high-value cue in the low/high compound, however, then they will prefer the low/high compound in both simple and compound probe trials, and vice versa if they allocate more attention to the low-value cue in the low/high compound. Choice behavior on probe trials therefore provides a measure of the distribution of attention to individual cues in the process of compound generalization.

The remaining 56 non-probe trials in this phase of the task were a mixture of different simple-versus-compound trials and compound-versus-compound trials (see Supplementary Materials, section S1 for details). This mixture of trial types was designed to yield a rich behavioral dataset that would allow us to fit computational models of the distinct psychological processes at play in compound generalization.

\subsection{Affect induction procedure}

We used a video-based procedure to induce either positive, neutral, or negative affect during the compound generalization phase of the task. Each of the three compound generalization blocks was preceded by a 90-second video with either positive, neutral, or negative emotional content such that each participant viewed three videos with the same emotional valence. Self-report ratings of affective arousal and valence were collected using affective sliders (Betella \& Verschure, 2016) at the beginning and end of each of these blocks, that is, right after and right before seeing a video.

We selected a set of 9 videos (3 happy, 3 neutral, $3 \mathrm{sad}$ ) from several sources, including affect-induction videos used in previous research (Hewig et al., 2005; Gruber, Johnson, Oveis, \& Keltner, 2008; Schaefer, Nils, Sanchez, \& Philippot, 2010) as well as publicly available clips chosen especially for this study. We confirmed the utility of these videos in inducing the desired affective states in a separate validation study conducted on Amazon Mechanical Turk. Full details of the specific videos used, as well as the results of the validation study are provided in the Supplementary Materials (section S2).

To measure baseline compound generalization behavior in the positive affect and negative affect groups (i.e., behavior prior to the affect induction), participants in these two groups completed an additional compound generalization block prior to the affect induction. This additional baseline block comprised 32 trials (16 simple-probe trials and 16 compound-probe trials). Participants in the neutral affect group did not complete this baseline block, since we assumed that these participants' performance was unaffected by the (neutral) videos that they watched. Indeed, the neutral group showed a flat mood profile throughout the compound generalization phase (Figure $2 \mathrm{~B}$ ), and as such, we were able to treat task performance for participants in this group as being at 'baseline' affect levels throughout the task.

Since no affect manipulation was conducted during the learning phase of the task, all participants were at a baseline mood level during the acquisition of cue-reward contingencies. This ensured that between-groups differences in choice behavior during the compound generalization phase of the task were attributable to the effects of the affect induction on compound generalization itself, rather than any effects of affect on learning. This is important given a large body of work that has shown complex effects of affect on learning across a number of tasks (e.g., Brand, Reimer, \& Opwis, 2007; Nadler, Rabi, \& Minda, 2010). 


\subsection{Eye-tracking}

\subsubsection{Data collection}

Eye-tracking data were acquired using an infrared eye-tracker (SR Research EyeLink 1000 Plus), at a sampling rate of $500 \mathrm{~Hz}$. Fixation points were calibrated prior to the task, and to control within-experiment drift we used automatic drift-correction after each block to re-calibrate fixations to the center of the screen. Before stimuli appeared on each choice trial, participants were required to maintain fixation within 200 pixels of the center of the screen for 1 second (i.e., stimuli did not appear on the screen until this condition was met). This ensured that participants began every choice trial fixating at a location that was approximately equidistant from the two stimuli. Raw output files were analysed using NivLink, an open-source Python package for preprocessing EyeLink eye-tracking data developed in-house (available at https://github.com/nivlab/NivLink).

\subsubsection{AoI selection and quality control}

Each simple cue was associated with an elliptical or semi-elliptical area of interest (AoI) defined around the center of its corresponding planet stimulus (Supplemental Figure S2). The major axes of the ellipses were angled at 45 and - 45 degrees from the vertical for the left and right stimulus respectively. To account for the different visual properties of simple and compound stimuli, we used a larger ellipsis around the compound stimulus (exactly twice the area of the simple-cue ellipsis), and created cue AoIs by dividing the ellipsis in two along the minor axis (Supplementary Figure S2C and S2D). This procedure ensured that while each cue could be enclosed either in a full or half-ellipse, the total area of its associated AoI was held constant across stimulus configurations.

In principle, the center of each ellipsis should coincide with the center of each planet stimulus. However, eye-tracking studies suffer from significant post-calibration drift, which typically increases with the length of the experiment (Vadillo, Street, Beesley, \& Shanks, 2015). To mitigate this issue, the center of each ellipsis was manually determined blockwise in post-processing by two independent raters (DB and AR). As a quality control step, we excluded from subsequent eye-tracking data analyses those participants for whom inter-rater agreement was low. Specifically, we excluded subjects for whom mean inter-rater disagreement was more than 1.5 of the inter-quartile range (IQR) above the third quartile of overall disagreement (specifically, participants with a mean disagreement of more than 32 pixels; see Supplementary Figure S4A). For each participant we additionally computed the percentage of samples that fall outside a valid AoI, and excluded from the analysis participants for whom this metric was 1.5 IQR above the third quartile (for these participants, more than $70 \%$ of all fixations were outside a valid AoI; see Supplementary Figure S4B). This yielded an eye-tracking sample of $\mathrm{N}=33, \mathrm{~N}=32$ and $\mathrm{N}=24$ participants in the neutral, positive and negative groups respectively. See Supplementary Sections S6 and S7 for more information on preprocessing and quality-control protocols for eye-tracking data. 


\subsubsection{Relative looking time}

Previous work has shown that the relative looking time to each feature of a multidimensional stimulus is a reliable measure of participants' focus of attention during decisionmaking (Leong, Radulescu, et al., 2017). A similar measure has been recently used in a study exploring the relationship between uncertainty, choice and eye-gaze (Stojić, Orquin, Dayan, Dolan, \& Speekenbrink, 2020). While relative looking time does not account for within-trial dynamics of visual fixations (Krajbich et al., 2010; Krajbich \& Rangel, 2011; Krajbich et al., 2012; Callaway \& Griffiths, 2019), it does provides a reliable and interpretable metric of how much relative sensory evidence a participant has collected about one cue versus another. Relative looking time can thus be used to detect biases in value computation.

In our task, relative looking time can be taken as an index of the extent to which participants are weighing each of the cues on the display in their decision. This is particularly useful for measuring attentional biases to cues that have higher or lower value, which we hypothesized would be influenced by affect. To compute the relative weight of simple cues within a compound, we summed the durations of fixations to each cue, and divided by the total time spent looking at any of the cues on the screen. This normalization allowed us to interpret relative looking time as a trial-specific measure of attention to each cue.

Because we were only interested in the relative weighting of cues during value computation, we restricted the computation of relative looking time to the time window before the participant chose a planet.

\subsection{Data quality control}

Eleven participants ( $9.2 \%$ of the sample) were excluded from all analyses: seven due to failure to learn outcome contingencies (based on a one-tailed binomial test against chance, $\alpha=.05)$, one due to excessive choice of empty stimuli in the learning phase $(>25 \%)$, one due to incongruent response to the negative affect induction (i.e., laughter), and two because of computer error resulting in failure to save data. 25 additional participants (20.8\% of the sample) were excluded from analysis of eye-tracking data only, as a result of failure of eye-tracking data quality-control checks described above.

\subsection{Statistical analysis}

Initial analyses used mixed-effects models as implemented in the lme4 package (Bates, Mächler, Bolker, \& Walker, 2015) in R to analyse participants' self-reported mood, choice behavior, and relative looking time. Self-report mood data were analysed using linear mixed-effects analyses, and choice behavior and relative looking time were analysed using mixed-effects logistic regression analyses. Random effects were selected according to a maximal- to minimal-that-converges procedure (Meteyard \& Davies, 2020), and incorporated random intercepts for participants as well as per-participant random slopes for effects that were entirely within-participant (Barr, Levy, Scheepers, \& Tily, 2013). pvalues were calculated using either the Satterthwaite degrees of freedom approximation 
(for linear mixed-effects analyses; Luke, 2017) or the Wald $t$-to- $z$ test (for mixed-effects logistic regression analyses).

For relative looking-time data, we weighted each trial by the total duration of fixations within the AOI (see Figure S6). As such, trials for which we had more valid eye-tracking data were weighted more heavily, thereby accounting for trial-by-trial differences in the precision of the relative looking time measure. This ensured that, for instance, a trial for which the total fixation duration to valid cues was 1000 milliseconds was treated as more informative regarding the latent probability of fixating on different cues than a trial for which the total fixation duration to valid cues was 100 milliseconds.

To reflect the hypothesized ordinal relation between affect groups, the video induction was coded as positive $=1$, neutral $=0$, negative $=-1$. Compound-generalization block number was recoded as $0,1,2$, such that coefficient estimates for other predictors reflect the effect of that predictor in the first compound generalization block, and interactions of that predictor with block number reflect changes in the strength of the predictor's effect relative to the first compound generalization block. To ensure that the interpretation of the coefficient quantifying the effect of block number on behavior was equivalent across affect-induction groups, the additional baseline generalization block completed by participants in the positive/negative affect groups was not included in analyses of block-dependent changes. Covariates (i.e. per-participant sum scores for each self-report measure) were centered and scaled across participants prior to inclusion in models. For analyses of post-choice mining response times, response times were log-transformed. Trials with mining response times in excess of 2.5 seconds (less than 1 percent of all trials) were excluded from reaction time analyses.

All statistical analyses reported in this manuscript can be reproduced using code and data available in the project's online repository (https://osf.io/egw5c/).

\subsection{Computational modelling procedure}

To formally test competing hypotheses about the potential effects of affect on compound generalization, we formulated and fit a number of computational models to participants' choice behavior in the compound generalization phase of the task. These models shared a common choice architecture, which we describe below in its maximal form (i.e., including all parameters). All models that we tested are variants of this maximal model.

We fit models to data within a hierarchical Bayesian framework, using Hamiltonian Monte Carlo as implemented in Stan (Carpenter et al., 2017) to sample from the joint posterior of all parameters. Four separate chains with randomized start values each took 2750 samples from the posterior. The first 1500 samples from each chain were discarded to prevent dependence on start values. This led to a total of 5000 post-warmup samples from the joint posterior. $\hat{R}$ for all parameters was less than 1.1, indicating acceptable convergence between chains, and there were no divergent transitions in any chain. To optimize sampling speed, all participant-level parameters were drawn from group-level Gaussian distributions whose means and standard deviations were estimated from the data, and then reparameterized to their respective non-centered parameterization (i.e., all parameters were sampled separately from a unit normal before being transformed to the appropriate range). In particular, to prevent negative values, several parameters were 
transformed using the normal cumulative distribution function (CDF) to lie in a positive range (softmax $\beta$ : standard normal $\mathrm{CDF} \times 50$; decay parameter $\lambda$ and compound bonus parameter $\phi$ : standard normal $\mathrm{CDF} \times 10)$.

Model comparison was performed using the Widely Applicable Information Criterion (WAIC; Watanabe, 2010), a statistic for comparing models fit with hierarchical Bayesian methods. The WAIC selects models according to their goodness-of-fit to data, minus a penalty for the model's effective complexity (estimated as variance of log-likelihood across posterior samples; Gelman, Hwang, \& Vehtari, 2014). We calculated the difference in WAIC between all models and the best-fitting model, and regarded the best-fitting model as credibly better than its competitors if the standard error of this difference did not overlap with zero. Ties were broken by selecting the model with fewer parameters (as a coarse proxy for model complexity).

\subsubsection{Overview of models}

We denote the value of a stimulus (i.e., a planet) by $V$ and the value of a cue (i.e., a rune image within a planet) by $v$. The reward-value $v$ of cues was defined as the true generative reward probability for each cue (i.e., $0.25,0.5$, or 0.75 ), under the assumption that cue values were learned correctly during the learning phase. For simple stimuli (those comprising only a single cue), the value of the stimulus was identical to the value of its constituent cue:

$$
V(\text { simple })=v_{\text {cue }}
$$

By contrast, the value of a compound stimulus was assumed to be a weighted linear sum of the values of the two cues comprising it:

$$
V(\text { compound })=\phi \cdot \sum_{j=1}^{2} A_{j} \cdot v_{j}
$$

where $A_{j}$ is the attention weight assigned to cue $j$ and $\phi$ is a scaling parameter that captures the general tendency for compound stimuli to be treated as more valuable $(\phi>$ 1 ) or less valuable $(\phi<1)$ than simple stimuli. In this case, $\phi=1$ corresponds to weighted averaging of simple cue values (as predicted by configural theories of compound generalization; e.g. Pearce, 1987), whereas $\phi=2$ (combined with equal attention to both cues, $A_{1}=A_{2}=0.5$ ) would better correspond to summation of simple cue values, as predicted by elemental theories of compound generalization (e.g., Rescorla \& Wagner, 1972).

Within a compound, the attention weight $A_{j}$ of cue $j$ was proportional to the rewardvalue of cue $j$ (weighted according to the $\alpha_{V}$ parameter), as well as to the certainty of cue $j$ (weighted according to the $\alpha_{C}$ parameter). Attention weights were then normalised across cues to ensure that the sum of attention weights for all cues within a single stimulus was 1:

$$
A_{j}=\frac{\exp \left(\alpha_{V} \cdot v_{j}+\alpha_{C} \cdot\left|v_{j}-0.5\right|\right)}{\sum_{k=1}^{N} \exp \left(\alpha_{V} \cdot v_{k}+\alpha_{C} \cdot\left|v_{k}-0.5\right|\right)}
$$

Here, $\alpha_{V}$ is a parameter that controls the degree to which attention is biased towards higher-value compounds (when $\alpha_{0}>0$, attention is drawn to higher-value cues; when 
$\alpha_{0}<0$, attention is drawn to lower-value cues) and $\alpha_{C}$ is a parameter that controls the degree to which attention is biased towards more certain cues (when $\alpha_{C}>0$, attention is drawn to the more-certain low and high cues; when $\alpha_{C}<0$, attention is drawn to the less-certain medium cue). In particular, cue certainty was calculated as the absolute difference between the reward probability of the cue and 0.5, such that a cue associated with a reward probability 0.5 was treated as maximally uncertain and cues associated with reward probabilities of 0.25 or 0.75 were treated as equally certain ${ }^{2}$. Variable attention to cue certainty allowed the model to flexibly account for the possibility that more predictive cues might either attract more attention (Mackintosh, 1975) or less attention (Pearce \& Hall, 1980) than less predictive cues.

Lastly, we assumed that choices were generated by a softmax function:

$$
\operatorname{Pr}(a=i)=\frac{\exp (\beta \cdot V(i))}{\sum_{x=1}^{2} \exp (\beta \cdot V(x))}
$$

where $i$ denotes the chosen stimulus, $X$ is the set of available stimuli, and $\beta$ is an inverse temperature parameter that controls the stochasticity of choices.

We selected the best-fitting computational model using a two-step procedure: in step 1 , we identified the model that provided the best fit to compound-generalization choices in the absence of an affect induction (i.e., all compound generalization choices for participants in the neutral affect group, and choices from the baseline block for participants in the positive- and negative-affect groups). In step 2, we tested which parameters of the best-fitting model were modulated by the affect induction itself (step 2 models were fit to all trials in the compound generalization phase from all affect-induction groups).

For each Step 2 model, we estimated two variants: one in which the effect of affect on parameters was constant across the three compound generalization blocks, and one in which the effect of affect weakened between blocks according to a power-law decay function. For an affect-related change in $\alpha_{V}$, for instance, the effective parameter value in a given block was computed as

$$
\alpha_{V}(\text { effective })=\alpha_{V}+\Delta \alpha_{V} \times \lambda^{\text {block }} \times \text { Affect }
$$

Here $\lambda$ is a block-wise decay parameter; when $\lambda=1$, there is no decay in the strength of affect-related parameter changes from one block to the next; by contrast, when $\lambda=0$, affect only influences parameters in the first compound generalization block (since $0^{0}=1$ ) and has no effect in later blocks. In Equation 7, the parameter $\Delta \alpha_{V}$ therefore quantifies the affect-related modulation of $\alpha_{V}$ in the first compound generalization block. A single $\lambda$ parameter was applied equally to all affect-related changes in each parameter, and $\lambda$ was estimated as a subject-level draw from a single group-level Gaussian distribution with a mean and standard deviation estimated from the data.

\footnotetext{
${ }^{2}$ Since there were only three reward probabilities in the present study, and since probabilities were symmetric around 0.5 , alternative uncertainty functions such as Shannon entropy would make similar predictions for our data.
} 


\section{References}

Anderson, B. A., Laurent, P. A., \& Yantis, S. (2012). Generalization of value-based attentional priority. Visual Cognition, 20(6), 647-658.

Aylward, J., Hales, C., Robinson, E., \& Robinson, O. J. (2020). Translating a rodent measure of negative bias into humans: The impact of induced anxiety and unmedicated mood and anxiety disorders. Psychological Medicine, 50(2), 237-246. doi: $10.1017 /$ S0033291718004117

Barr, D. J., Levy, R., Scheepers, C., \& Tily, H. J. (2013). Random effects structure for confirmatory hypothesis testing: Keep it maximal. Journal of Memory and Language, 68(3), 255-278.

Bates, D., Mächler, M., Bolker, B. M., \& Walker, S. C. (2015). Fitting linear mixed-effects models using lme4. Journal of Statistical Software, 67(1), 1-48.

Becker, M. W., \& Leinenger, M. (2011). Attentional selection is biased toward moodcongruent stimuli. Emotion, 11(5), 1248-1254. doi: 10.1037/a0023524

Bennett, D., Davidson, G., \& Niv, Y. (2021). A model of mood as integrated advantage. Psychological Review. doi: 10.1037/rev0000294

Bennett, D., \& Niv, Y. (2020). Opening Burton's clock: psychiatric insights from computational cognitive models. In D. Poeppel, G. R. Mangun, \& M. S. Gazzaniga (Eds.), (pp. 439-449). Cambridge, MA: The MIT Press. doi: 10.31234/osf.io/y2vzu

Bennett, D., Silverstein, S. M., \& Niv, Y. (2019). The two cultures of computational psychiatry. JAMA Psychiatry, 76(6), 563-564.

Betella, A., \& Verschure, P. F. (2016). The affective slider: A digital self-assessment scale for the measurement of human emotions. PLOS one, 11 (2), e0148037.

Bower, G. H. (1981). Mood and memory. American Psychologist, 36(2), 129-148.

Brainard, D. H. (1997). The psychophysics toolbox. Spatial Vision, 10(4), 433-436.

Brand, S., Reimer, T., \& Opwis, K. (2007). How do we learn in a negative mood? effects of a negative mood on transfer and learning. Learning and instruction, 17(1), $1-16$.

Callaway, F., \& Griffiths, T. (2019). Attention in value-based choice as optimal sequential sampling.

Carpenter, B., Gelman, A., Hoffman, M. D., Lee, D., Goodrich, B., Betancourt, M., .. Riddell, A. (2017). Stan: A probabilistic programming language. Journal of Statistical Software, 76(1).

Chun, M. M., Golomb, J. D., \& Turk-Browne, N. B. (2011). A taxonomy of external and internal attention. Annual Review of Psychology, 62(1), 73-101. doi: 10.1146/ annurev.psych.093008.100427

Daniel-Watanabe, L., McLaughlin, M., Gormley, S., \& Robinson, O. J. (2020). Association between a directly translated cognitive measure of negative bias and selfreported psychiatric symptoms. Biological Psychiatry: Cognitive Neuroscience and Neuroimaging. doi: 10.1016/j.bpsc.2020.02.010

Depue, R. A., Slater, J. F., Wolfstetter-Kausch, H., Klein, D., Goplerud, E., \& Farr, D. (1981). A behavioral paradigm for identifying persons at risk for bipolar depressive disorder: A conceptual framework and five validation studies. Journal of Abnormal Psychology, 90(5), 381-437. 
Duncan, K., Doll, B. B., Daw, N. D., \& Shohamy, D. (2018). More than the sum of its parts: A role for the hippocampus in configural reinforcement learning. Neuron, 98(3), 645-657.

Eldar, E., Pessiglione, M., \& van Dillen, L. (2021). Positive affect as a computational mechanism. Current Opinion in Behavioral Sciences, 39, 52-57.

Eldar, E., Rutledge, R. B., Dolan, R. J., \& Niv, Y. (2016). Mood as representation of momentum. Trends in cognitive sciences, 20(1), 15-24.

Erez, A., \& Isen, A. M. (2002). The influence of positive affect on the components of expectancy motivation. Journal of Applied Psychology, 87(6), 1055-1067. doi: 10.1037/0021-9010.87.6.1055

Eysenck, M. W., Mogg, K., May, J., Richards, A., \& Mathews, A. (1991). Bias in interpretation of ambiguous sentences related to threat in anxiety. Journal of Abnormal Psychology, 100 (2), 144-150.

Fredrickson, B. L. (1998). What good are positive emotions? Review of General Psychology, 2(3), 300-319.

Fredrickson, B. L. (2004). The broaden-and-build theory of positive emotions. Philosophical Transactions of the Royal Society of London. Series B: Biological Sciences, 359(1449), 1367-1377.

Gable, P., \& Harmon-Jones, E. (2010). The motivational dimensional model of affect: Implications for breadth of attention, memory, and cognitive categorisation. Cognition and Emotion, 24(2), 322-337.

Gelman, A., Hwang, J., \& Vehtari, A. (2014). Understanding predictive information criteria for bayesian models. Statistics and Computing, 24(6), 997-1016.

Gotlib, I. H., \& Joormann, J. (2010). Cognition and depression: current status and future directions. Annual review of clinical psychology, 6, 285-312.

Gruber, J., Johnson, S. L., Oveis, C., \& Keltner, D. (2008). Risk for mania and positive emotional responding: Too much of a good thing? Emotion, 8(1), 23-33.

Gruber, J., Maclaine, E., Avard, E., Purcell, J., Cooper, G., Tobias, M., ... others (2021). Associations between hypomania proneness and attentional bias to happy, but not angry or fearful, faces in emerging adults. Cognition and Emotion, 35(1), 1-7.

Harding, E. J., Paul, E. S., \& Mendl, M. (2004, January). Cognitive bias and affective state. Nature, 427(6972), 312-312. doi: 10.1038/427312a

Hewig, J., Hagemann, D., Seifert, J., Gollwitzer, M., Naumann, E., \& Bartussek, D. (2005). A revised film set for the induction of basic emotions. Cognition and Emotion, 19(7), 1095-1109.

Hirsch, C., \& Mathews, A. (1997). Interpretative inferences when reading about emotional events. Behaviour Research and Therapy, 35(12), 1123-1132.

Holland, P. C. (1991). Transfer of control in ambiguous discriminations. Journal of Experimental Psychology: Animal Behavior Processes, 17(3), 231.

Itti, L., \& Baldi, P. (2009). Bayesian surprise attracts human attention. Vision Research, $49(10), 1295-1306$.

Joffily, M., \& Coricelli, G. (2013). Emotional valence and the free-energy principle. PLOS Computational Biology, 9(6), e1003094.

Johnson, E. J., \& Tversky, A. (1983). Affect, generalization, and the perception of risk. 
Journal of Personality and Social Psychology, 45(1), 20-31.

Johnson, S. L. (2005). Mania and dysregulation in goal pursuit: A review. Clinical Psychology Review, 25(2), 241-262. doi: 10.1016/j.cpr.2004.11.002

Johnson, S. L., Freeman, M. A., \& Staudenmaier, P. J. (2015). Mania risk, overconfidence, and ambition. Journal of Social and Clinical Psychology, 34(7), 611-621.

Kehoe, E. J., Horne, A. J., Horne, P. S., \& Macrae, M. (1994). Summation and configuration between and within sensory modalities in classical conditioning of the rabbit. Animal Learning \&3 Behavior, 22(1), 19-26.

Koster, E. H. W., De Raedt, R., Goeleven, E., Franck, E., \& Crombez, G. (2005). Moodcongruent attentional bias in dysphoria: Maintained attention to and impaired disengagement from negative information. Emotion, 5(4), 446-455. doi: 10.1037/ 1528-3542.5.4.446

Koster, E. H. W., De Raedt, R., Leyman, L., \& De Lissnyder, E. (2010). Moodcongruent attention and memory bias in dysphoria: Exploring the coherence among information-processing biases. Behaviour Research and Therapy, 48(3), 219-225. doi: 10.1016/j.brat.2009.11.004

Krajbich, I., Armel, C., \& Rangel, A. (2010). Visual fixations and the computation and comparison of value in simple choice. Nature Neuroscience, 13(10), 1292.

Krajbich, I., Lu, D., Camerer, C., \& Rangel, A. (2012). The attentional drift-diffusion model extends to simple purchasing decisions. Frontiers in psychology, 3, 193.

Krajbich, I., \& Rangel, A. (2011). Multialternative drift-diffusion model predicts the relationship between visual fixations and choice in value-based decisions. Proceedings of the National Academy of Sciences, 108(33), 13852-13857.

Kruschke, J. K. (1992). ALCOVE: An exemplar-based connectionist model of category learning. Psychological Review, 99(1), 22-44.

Kruschke, J. K. (2001). Toward a unified model of attention in associative learning. Journal of Mathematical Psychology, 45 (6), 812-863. doi: 10.1006/jmps.2000.1354

Leong, Y. C., Radulescu, A., Daniel, R., DeWoskin, V., \& Niv, Y. (2017). Dynamic interaction between reinforcement learning and attention in multidimensional environments. Neuron, 93(2), 451-463.

Le Pelley, M. E., Mitchell, C. J., Beesley, T., George, D. N., \& Wills, A. J. (2016). Attention and associative learning in humans: An integrative review. Psychological Bulletin, 142(10), 1111-1140. doi: 10.1037/bul0000064

Luke, S. G. (2017). Evaluating significance in linear mixed-effects models in r. Behavior Research Methods, 49(4), 1494-1502.

Mackintosh, N. J. (1975). A theory of attention: Variations in the associability of stimuli with reinforcement. Psychological Review, 82(4), 276--298.

MacLeod, A. K. (1996, January). Affect, emotional disorder, and future-directed thinking. Cognition \& Emotion, 10(1), 69-86. doi: 10.1080/026999396380394

MacLeod, A. K., Tata, P., Kentish, J., Carroll, F., \& Hunter, E. (1997). Anxiety, depression, and explanation-based pessimism for future positive and negative events. , 4 , 10.

MacLeod, C., Mathews, A., \& Tata, P. (1986). Attentional bias in emotional disorders. Journal of Abnormal Psychology, 95(1), 15-20. 
Marshall, G. N., Wortman, C. B., Kusulas, J. W., Hervig, L. K., \& Vickers, R. R. (1992). Distinguishing optimism from pessimism: relations to fundamental dimensions of mood and personality. Journal of Personality and Social Psychology, 62(6), 10671074.

Mayer, J. D., Gaschke, Y. N., Braverman, D. L., \& Evans, T. W. (1992). Mood-congruent judgment is a general effect. Journal of Personality and Social Psychology, 63(1), 119.

McLaren, I. P. L., \& Mackintosh, N. J. (2002). Associative learning and elemental representation: II. Generalization and discrimination. Animal Learning $\mathcal{E}$ Behavior, 30(3), 177-200. doi: 10.3758/BF03192828

Melchers, K. G., Shanks, D. R., \& Lachnit, H. (2008). Stimulus coding in human associative learning: Flexible representations of parts and wholes. Behavioural Processes, 77(3), 413-427. doi: 10.1016/j.beproc.2007.09.013

Mendl, M., Burman, O. H., Parker, R. M., \& Paul, E. S. (2009). Cognitive bias as an indicator of animal emotion and welfare: Emerging evidence and underlying mechanisms. Applied Animal Behaviour Science, 118(3-4), 161-181.

Meteyard, L., \& Davies, R. A. (2020). Best practice guidance for linear mixed-effects models in psychological science. Journal of Memory and Language, 112, 104092. doi: $10.1016 /$ j.jml.2020.104092

Miranda, R., \& Mennin, D. S. (2007). Depression, generalized anxiety disorder, and certainty in pessimistic predictions about the future. Cognitive Therapy and Research, $31(1), 71-82$.

Moore, S. C., \& Oaksford, M. (2002). An informational value for mood: Negative mood biases attention to global information in a probabilistic classification task. In Emotional Cognition: From Brain to Behaviour (pp. 221-243). Amsterdam/Philadelphia: John Benjamins Publishing Company.

Nadler, R. T., Rabi, R., \& Minda, J. P. (2010). Better mood and better performance: Learning rule-described categories is enhanced by positive mood. Psychological Science, 21(12), 1770-1776.

Niv, Y., Daniel, R., Geana, A., Gershman, S. J., Leong, Y. C., Radulescu, A., \& Wilson, R. C. (2015). Reinforcement learning in multidimensional environments relies on attention mechanisms. Journal of Neuroscience, 35(21), 8145-8157. doi: 10.1523/ JNEUROSCI.2978-14.2015

Nosofsky, R. M. (1986). Attention, similarity, and the identification-categorization relationship. Journal of Experimental Psychology: General, 115(1), 39-57.

Paul, K., \& Pourtois, G. (2017). Mood congruent tuning of reward expectation in positive mood: Evidence from FRN and theta modulations. Social Cognitive and Affective Neuroscience, 12(5), 765-774. doi: 10.1093/scan/nsx010

Pearce, J. M. (1987). A model for stimulus generalization in Pavlovian conditioning. Psychological Review, 94(1), 61-73.

Pearce, J. M., \& Hall, G. (1980). A model for Pavlovian learning: variations in the effectiveness of conditioned but not of unconditioned stimuli. Psychological Review, $87(6), 532-552$.

Poder, E. (2006). Crowding, feature integration, and two kinds of "attention". Journal 
of Vision, 6(2), 7 .

Radulescu, A., \& Niv, Y. (2019). State representation in mental illness. Current opinion in neurobiology, 55, 160-166.

Radulescu, A., Niv, Y., \& Ballard, I. (2019). Holistic reinforcement learning: the role of structure and attention. Trends in Cognitive Sciences, 23(4), 278-292.

Raila, H., Scholl, B. J., \& Gruber, J. (2015). Seeing the world through rose-colored glasses: People who are happy and satisfied with life preferentially attend to positive stimuli. Emotion, 15(4), 449-462.

Ransom, M., Fazelpour, S., Markovic, J., Kryklywy, J., Thompson, E. T., \& Todd, R. M. (2020). Affect-biased attention and predictive processing. Cognition, 203, 104370.

Rescorla, R. A. (1976). Stimulus generalization: Some predictions from a model of Pavlovian conditioning. Journal of Experimental Psychology: Animal Behavior Processes, 2(1), 88-96.

Rescorla, R. A., \& Wagner, A. R. (1972). A theory of Pavlovian conditioning: Variations in the effectiveness of reinforcement and nonreinforcement. In Classical Conditioning: Current Research and Theory (Vol. 2, pp. 64-99). New York: AppletonCentury-Crofts.

Rief, W., Glombiewski, J. A., Gollwitzer, M., Schubö, A., Schwarting, R., \& Thorwart, A. (2015). Expectancies as core features of mental disorders:. Current Opinion in Psychiatry, 28(5), 378-385. doi: 10.1097/YCO.0000000000000184

Rouhani, N., \& Niv, Y. (2019). Depressive symptoms bias the prediction-error enhancement of memory towards negative events in reinforcement learning. Psychopharmacology, 236 (8), 2425-2435.

Saavedra, M. A. (1975). Pavlovian compound conditioning in the rabbit. Learning and Motivation, 6(3), 314-326.

Schaefer, A., Nils, F., Sanchez, X., \& Philippot, P. (2010). Assessing the effectiveness of a large database of emotion-eliciting films: A new tool for emotion researchers. Cognition and Emotion, $24(7), 1153-1172$.

Scheier, M. F., Carver, C. S., \& Bridges, M. W. (2001). Optimism, pessimism, and psychological well-being. In E. C. Chang (Ed.), Optimism 83 pessimism: Implications for theory, research, and practice. (pp. 189-216). Washington: American Psychological Association. doi: 10.1037/10385-009

Schönfelder, S., Langer, J., Schneider, E. E., \& Wessa, M. (2017). Mania risk is characterized by an aberrant optimistic update bias for positive life events. Journal of Affective Disorders, 218, 313-321.

Schulz, E., Bhui, R., Love, B. C., Brier, B., Todd, M. T., \& Gershman, S. J. (2019). Structured, uncertainty-driven exploration in real-world consumer choice. Proceedings of the National Academy of Sciences, 116(28), 13903-13908. doi: 10.1073/ pnas. 1821028116

Sedikides, C. (1992). Mood as a determinant of attentional focus. Cognition \& Emotion, $6(2), 129-148$.

Segerstrom, S. C. (2001). Optimism and attentional bias for negative and positive stimuli. Personality and Social Psychology Bulletin, 27(10), 1334-1343. doi: 10 $.1177 / 01461672012710009$ 
Shanks, D. R., \& Darby, R. J. (1998). Feature and rule-based generalization in human associative learning. Journal of Experimental Psychology: Animal Behavior Processes, 24(4), 405-415.

Shepard, R. N. (1987). Toward a universal law of generalization for psychological science. Science, 237(4820), 1317-1323.

Soto, F. A., Gershman, S. J., \& Niv, Y. (2014). Explaining compound generalization in associative and causal learning through rational principles of dimensional generalization. Psychological Review, 121(3), 526-558. doi: 10.1037/a0037018

Stojić, H., Orquin, J. L., Dayan, P., Dolan, R. J., \& Speekenbrink, M. (2020). Uncertainty in learning, choice, and visual fixation. Proceedings of the National Academy of Sciences, 117(6), 3291-3300.

Tamir, M., \& Robinson, M. D. (2007). The happy spotlight: Positive mood and selective attention to rewarding information. Personality and Social Psychology Bulletin, $33(8), 1124-1136$.

Vadillo, M. A., Street, C. N., Beesley, T., \& Shanks, D. R. (2015). A simple algorithm for the offline recalibration of eye-tracking data through best-fitting linear transformation. Behavior Research Methods, 47(4), 1365-1376.

Vater, C., Kredel, R., \& Hossner, E.-J. (2017). Disentangling vision and attention in multiple-object tracking: How crowding and collisions affect gaze anchoring and dual-task performance. Journal of Vision, 17(5), 21.

Vinckier, F., Rigoux, L., Oudiette, D., \& Pessiglione, M. (2018). Neuro-computational account of how mood fluctuations arise and affect decision making. Nature communications, 9(1), 1-12.

Wadlinger, H. A., \& Isaacowitz, D. M. (2006). Positive mood broadens visual attention to positive stimuli. Motivation and Emotion, 30(1), 87-99.

Wagner, A. R. (2008). Evolution of an elemental theory of Pavlovian conditioning. Learning \& Behavior, 36(3), 253-265. doi: 10.3758/LB.36.3.253

Watanabe, S. (2010). Asymptotic equivalence of Bayes cross validation and widely applicable information criterion in singular learning theory. Journal of Machine Learning Research, 11, 3571-3594.

Watson, D., Clark, L. A., \& Tellegen, A. (1988). Development and validation of brief measures of positive and negative affect: the PANAS scales. Journal of Personality and Social Psychology, 54(6), 1063-1070.

Whitney, D., \& Levi, D. M. (2011). Visual crowding: A fundamental limit on conscious perception and object recognition. Trends in Cognitive Sciences, 15(4), 160-168.

Wright, W. F., \& Bower, G. H. (1992). Mood effects on subjective probability assessment. Organizational Behavior and Human Decision Processes, 52(2), 276-291. doi: 10 .1016/0749-5978(92)90039-A

Zivot, M. T., Cohen, A. L., \& Kapucu, A. (2013). Modeling the effect of mood on dimensional attention during categorization. Emotion, 13(4), 703-710. doi: 10 $.1037 / \mathrm{a} 0031987$

Zorowitz, S., Momennejad, I., \& Daw, N. D. (2020). Anxiety, avoidance, and sequential evaluation. Computational Psychiatry, 4, 1-17. 